August 4, 2005

\title{
A Test Problem for Molecular Dynamics INTEGRATORS
}

\author{
P. F. Tupper \\ Department of Mathematics and Statistics, McGill University \\ Montréal, Québec, CANADA, H3A 2K6.
}

\begin{abstract}
We derive a test problem for evaluating the ability of time-stepping methods to preserve statistical properties of systems in molecular dynamics. We consider a family of deterministic systems consisting of a finite number of particles interacting on a compact interval. The particles are given random initial conditions and interact through instantaneous energy- and momentum-conserving collisions. As the number of particles, the particle density, and the mean particle speed go to infinity, the trajectory of a tracer particle is shown to converge to a stationary Gaussian stochastic process. We approximate this system by one described by a system of ordinary differential equations and provide numerical evidence that it converges to the same stochastic process. We simulate the latter system with a variety of numerical integrators, including the symplectic Euler method, a fourth-order Runge-Kutta method, and an energy-conserving step-and-project method. We assess the methods' ability to recapture the system's limiting statistics and observe that symplectic Euler performs significantly better than the others for comparable computational expense.
\end{abstract}

\section{Introduction}

In the field of molecular dynamics, researchers use numerical integrators to approximate the motion of systems of particles. They integrate over long periods of time and extract statistical information from the computed trajectories. This, in turn, can be used to determine macroscopic properties of the system. For optimal efficiency, the researchers integrate using as long a step-length as possible while still maintaining the stability of the computed 
solution. In this regime trajectories are not computed accurately. Nevertheless, it is observed that statistical features of solutions are maintained in some circumstances. (See [1],[4].)

One possible explanation for this phenomenon is the existence of an underlying stochastic process [19]. Suppose that the trajectories of the deterministic process approximate some stochastic process in the sense of distribution. If we use a numerical method whose trajectories also approximate the same stochastic process, then the numerical solution will have similar statistical features to the original system, even though there is no path-wise agreement.

The goal of this paper is to construct a test case for this situation. We seek a deterministic system having a component of its trajectory that approximates a well-understood stochastic process. Once given such a system, we can use it to test numerical integrators. We integrate the system with a numerical integrator using step-lengths that do not resolve the trajectories correctly. Then we can investigate how accurately these computed solutions reproduce the statistical features of the underlying stochastic process.

Our construction is inspired by a 1968 paper of Spitzer [20] that provides an example of a sequence of deterministic systems whose trajectories converge to a stochastic process. Building on the previous work of Harris [12], he shows that Brownian motion can be obtained as the limit of a sequence of deterministic processes on the real line with random initial conditions. His construction consists of placing point particles on the real line according to a Poisson distribution. Each particle is assigned a random velocity independently of the other particles. The particles are allowed to move, so that they interact through energy- and momentum-conserving collisions: i.e., whenever two particles meet, there is an instantaneous collision in which they exchange velocities. A single particle is placed at the origin and its subsequent trajectory observed. Spitzer proves that with an appropriate scaling of the variables, the path of this tracer particle converges weakly to standard Brownian motion.

There are two difficulties with using this system for our investigations. The first is that since it is infinite in extent, it is impossible to simulate it completely on a computer. As a way of avoiding this difficulty, in Section 2 we introduce a finite counterpart to Spitzer's set-up. We describe a sequence of systems each consisting of a finite number of particles interacting on a compact segment of the real line. In Sections 3 and 4 we prove that as the number of particles goes to infinity, the trajectory of a tracer particle will 
converge to a stationary Gaussian process with a known correlation function.

The second difficulty is that neither Spitzer's system nor the system we present in Section 2 are described purely in terms of ordinary differential equations, since the inter-particle collisions are instantaneous. Thus we cannot use these systems as test problems for numerical integrators without dealing with the issues of collision detection. However, we show in Section 5 how we can approximate the non-differentiable flow of these systems with the flow of a differential equation by replacing the hard, instantaneous collisions of the particles with collisions mediated by a soft potential. This system approximates the non-differentiable system in the limit as the stiffness of the inter-particle forces goes to infinity. If we allow both the number of particles and the inter-particle stiffness to go to infinity with a particular scaling, we conjecture that the tracer particle's trajectory converges to the same Gaussian process. We show the results of numerical experiments that support this statement.

In Section 6 we use the family of systems introduced in Section 5 as a test problem for a variety of time-stepping methods. We apply five different numerical methods to the system: symplectic Euler, backward Euler, forward Euler, a fourth-order Runge-Kutta method, and an energy-conserving step-and-project method. We observe that, of these methods, only symplectic Euler and the step-and-project method are able to even approximatley reproduce statistics in the limiting case with reasonable step sizes. The latter energy-conserving method is more costly and is not able to take longer steps than symplectic Euler without damaging its ability to recover the stochastic limit. We conclude that, for this test problem, the symplectic Euler method is clearly the superior method out of those considered. This coincides with computational practice in molecular dynamics, where use of the Verlet integrator (a version of symplectic Euler) is ubiquitous [1].

Though our interest in simulation has led us to consider finite systems, other researchers have continued with Spitzer's ideas in [20] in different directions. One possibility is to allow the mass of the tracer to differ from the mass of the other particles. In [13] Holley proves that with such a scaling the trajectory of the tracer particle weakly converges to the Ornstein-Uhlenbeck process. In [16] Mürmann takes this result further by proving a similar result when the collisions between the particles do not occur instantly but are mediated by a soft potential.

Other work has had the same motivation as this one - to create suitable test problems for molecular dynamics simulations - but has considered a 
different class of problems. The papers [21], [11], [15] have rigorously shown stochastic limiting behaviour for the Ford-Kac heat-bath model [8]. Like the model in the present paper, a sequence of Hamiltonian systems with random initial conditions and increasing numbers of particles is considered. In the infinite particle limit the trajectory of the tracer particle is shown to converge to the solution of a stochastic differential equation. This construction has been used to analyse numerical integrators in [3] and more sophisticated algorithms in [14].

\section{Particle Systems on a Compact Interval}

In this section we describe a sequence of particle systems on the interval $[-1,1]$. For each odd positive integer $n$, the system will consist of $n$ interacting particles (contrary to the convention in the interacting particle literature). We let the tracer particle be the "median" particle in the interval, that is, the one with an equal number of particles on either side of it. When we scale the interval by a factor of $n^{1 / 2}$ we will see that the trajectory of this tracer particle will converge to a Gaussian random process.

Let $q_{i}, i=1, \ldots, n$ be i.i.d. random variables, each uniformly distributed on $[-1,1]$. These are the initial positions of the $n$ particles. We give the particles i.i.d. velocities $p_{i}$ according to a distribution with a probability density $f$. We make the following assumptions on $f$.

Assumptions 2.1 The probability density $f$ satisfies

(i) $f$ is symmetric: $f(-p)=f(p)$.

(ii) $f$ is $L_{1}$ and nontrivial: $0<\mathbb{E}\left|p_{i}\right|<\infty$.

(iii) $f$ is compactly supported: $\mathbb{P}\left\{\left|p_{i}\right|>V_{\max }\right\}=0$ for some $V_{\max }$.

Note that the third condition implies that $f$ is $L_{1}$. However, we leave the assumptions in this form since some results will be proven without using condition (iii).

Given these initial conditions, we allow the particles to move according to the following rules. Particles move at constant velocity until they encounter either another particle or one of the barriers at -1 or 1 . If two particles collide, they merely exchange velocities. If a particle hits a wall, it reverses its velocity. These rules are a natural consequence of assuming that the total energy of the system is conserved and that when two particles collide, their total momentum is conserved. With these rules for motion we designate the 
position at time $t$ of the particle starting at $q_{i}$ by $x_{i}(t)$. Note that the order of the particles is unchanged in time. By this we mean that if $q_{i}<q_{j}$ then $x_{i}(t) \leq x_{j}(t)$ for all $t \geq 0$. Choose $i_{\text {med }}$ so that $q_{i_{m e d}}$ is the median of the $\left\{q_{i}\right\}$. It follows that $x_{i_{\text {med }}}(t)$ is the median of the $\left\{x_{i}(t)\right\}$ for all $t$. We choose this particle to be our tracer particle and define $x_{\text {med }}(t):=x_{i_{\text {med }}}(t)$ for $t \geq 0$.

If we multiply the positions of all of the particles over time by $n^{1 / 2}$, we obtain a system of $n$ particles interacting over the interval $\left[-n^{1 / 2}, n^{1 / 2}\right]$, with an average particle speed of $n^{1 / 2} \mathbb{E}\left|p_{i}\right|$. We will denote the position of the tracer particle with this scaling by $U_{n}$, so that $U_{n}(t)=n^{1 / 2} x_{m e d}(t)$ for $t \geq 0$.

In order to get a more convenient representation of the motion of the tracer particle, we now describe a different, but related, set of rules of motion. The rules of motion are the same as before except that the particles do not interact with each other, but only the walls. Thus when the trajectories of two particles intersect, the particles merely pass through each other. Under this set of rules, we designate the position of the particle starting at $q_{i}$ by $y_{i}(t)$, for $t \geq 0$. There is a simple expression for $y_{i}(t)$. If there were no walls, the particle's position at time $t$ would be $q_{i}+t p_{i}$. The effect of the walls is to "fold" the particle's trajectory back into the interval $[-1,1]$. This is accomplished by a function $G$ such that

$$
y_{i}(t)=G\left(q_{i}+t p_{i}\right)
$$

$G$ is the periodic function with period 4 such that

$$
G(x)=\left\{\begin{array}{rr}
x, & -1 \leq x \leq 1 \\
2-x, & 1 \leq x \leq 3 .
\end{array}\right.
$$

There is a relationship between the two sets of trajectories, $\left\{x_{i}(t)\right\}$ and $\left\{y_{i}(t)\right\}$. At any point in time, the set of positions the particles take is the same under the two different rules of motion. Since the position of the tracer particle at any time is given by the median of all the $x_{i}(t)$, its position is also given by the median of all the $y_{i}(t)$. So the trajectory of the tracer particle is given by

$$
U_{n}(t):=n^{1 / 2} x_{\text {med }}(t)=n^{1 / 2} \operatorname{med}_{i=1 \ldots n}\left(y_{i}(t)\right)=n^{1 / 2} \operatorname{med}_{i=1 \ldots n}\left(G\left(q_{i}+t p_{i}\right)\right) .
$$

This is a convenient representation, since $\left\{q_{i}\right\}_{i=1}^{n}$ and $\left\{p_{i}\right\}_{i=1}^{n}$ are independent random variables with known distributions. 


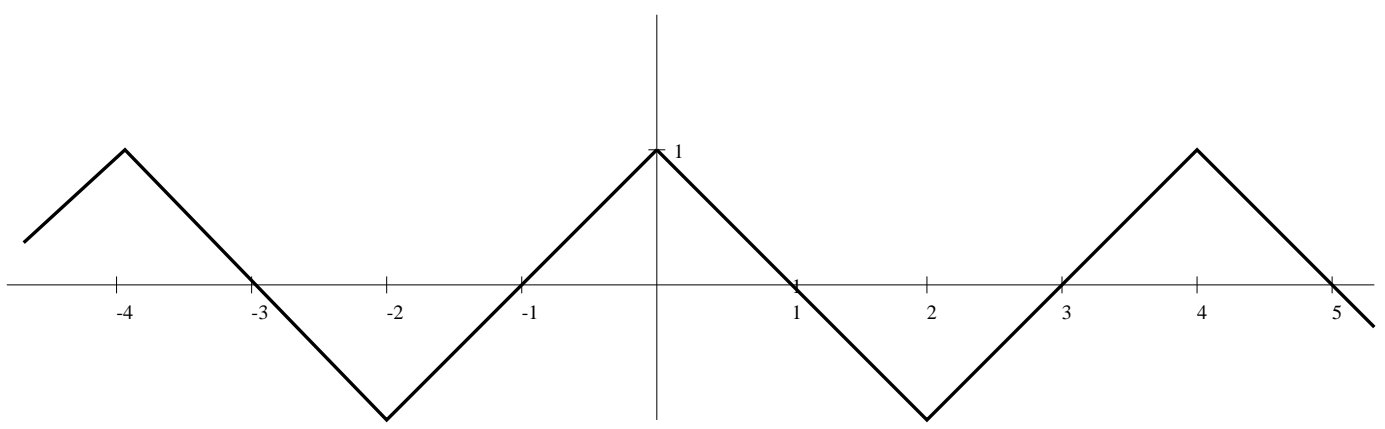

Figure 2.1: The function $H$.

It is straightforward to check that for each $i, y_{i}$ is a stationary stochastic process. As a consequence, both $x_{m e d}$ and $U_{n}$ are stationary processes as well. The distribution of $y_{i}(t)$ is uniform on $[-1,1]$ for all $t$.

We briefly review the concept of weak convergence in a topological space. Let $X$ be a topological space with random elements $U, U_{n}, n \geq 1$. We say that $U_{n}$ weakly converges to $U$, and write $U_{n} \Rightarrow U$, if for all bounded continuous functions $g: X \rightarrow \mathbb{R}, \mathbb{E} g\left(U_{n}\right) \rightarrow \mathbb{E} g(U)$ as $n \rightarrow \infty$. We will consider two examples of $X$ in this paper. The first is $C[0, T]$, the space of real-valued continuous functions on $[0, T]$ with the topology induced by the supremum norm. The second is $\mathbb{R}^{d}$ with the standard topology. See [2] for a thorough exposition of weak convergence.

We now present our main result. It states that as the number of particles $n$ goes to infinity, the scaled trajectory of the tracer particle will converge weakly to a Gaussian process in $C[0, T]$. This result is the basis for the numerical experiments performed later in the paper. For the statement of the main theorem, we define $H$ to be a periodic function with period 4 such that

$$
H(z)= \begin{cases}1-z, & 0 \leq z \leq 2 \\ z-3, & 2 \leq z \leq 4\end{cases}
$$

as shown in Figure 2.1.

Theorem 2.2 Let $\left\{q_{i}\right\}_{i \geq 1},\left\{p_{i}\right\}_{i \geq 1}$, be mutually independent i.i.d. sequences of random variables, where each $q_{i}$ is distributed uniformly on $[-1,1]$ and each $p_{i}$ is distributed with density $f$ satisfying Assumptions 2.1. For each odd positive integer $n$ let

$$
U_{n}(t):=n^{1 / 2} \operatorname{med}_{i=1 \ldots n}\left(G\left(q_{i}+t p_{i}\right)\right) .
$$



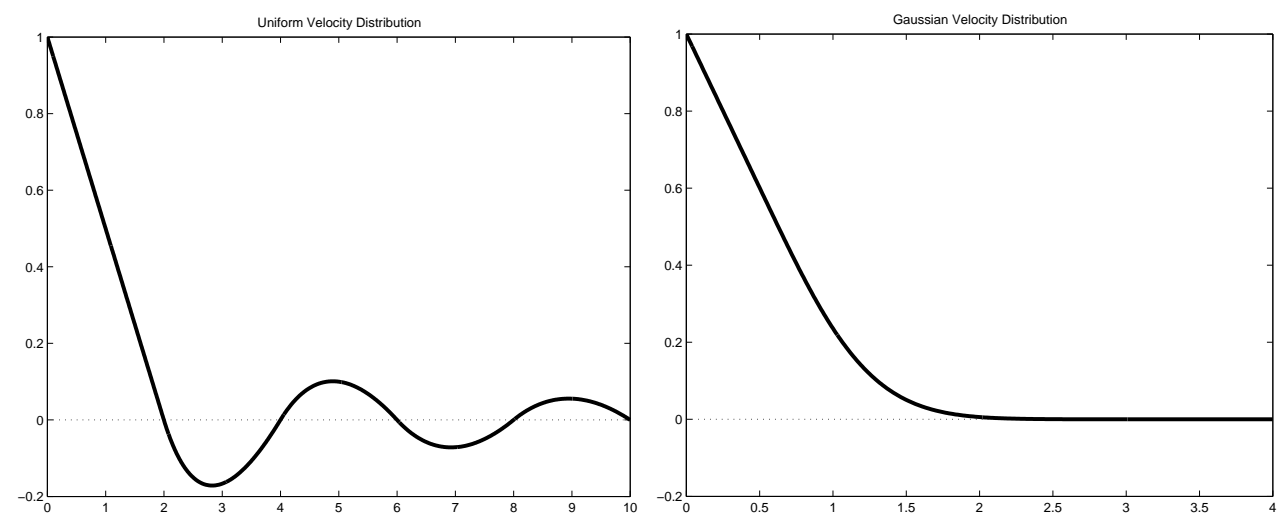

Figure 2.2: Covariance functions for two choices of the velocity distribution.

Then as $n \rightarrow \infty$,

$$
U_{n}(t) \Rightarrow U(t),
$$

where $U(t)$ is the stationary continuous mean-zero Gaussian process with covariance

$$
C(t):=\mathbb{E}[U(0) U(t)]=\int_{-\infty}^{\infty} H(p t) f(p) d p .
$$

The convergence is in the weak sense in $\mathrm{C}[0, T]$ for any $T>0$.

We note that we will have completely determined the limiting process since a mean-zero continuous Gaussian process is completely specified by its covariance function. Since our process is stationary we have

$$
\mathbb{E} U(s) U(t)=\mathbb{E} U(0) U(t-s)=C(t-s) .
$$

Equation 2.5 gives the covariance $C$ as it depends on the velocity density function $f$. In Figure 2.2 we show plots of $C$ vs $t$ for two choices of $f$. The first shows the case when velocities are chosen uniformly in $[-1,1]$; the second shows the case when they are chosen according to the standard Gaussian distribution. Alternatively, when we choose velocities to be either -1 or 1 , each with probability $1 / 2$, then $C=H$ defined by (2.4) and shown in Figure 2.1.

In each case, the covariance function gives information about the behaviour of the limiting stochastic process. The value $C(0)$ gives the variance of the process, which is constant in time. Values $C(t)$ for $t>0$ indicate the covariance between the position of the tracer particle at a given time and its 
position at $t$ time units later. For both the uniform $f$ and the Gaussian $f$, position at time $t$ decorrelates with position at time 0 as $t$ goes to infinity. In the former case, there are alternating periods of positive and negative correlation, whereas in the latter there is positive correlation for all times $t$. In the case of the \pm 1 velocity distribution there is no decay of correlation. This occurs because all the particles are moving at speed 1. Since the box has length 2, all particles return to where they were initially after every 4 time units. Thus both the trajectory of the tracer particle and the covariance function itself are periodic functions of period 4 .

The standard method for proving weak convergence of random processes in $C[0, T]$ is to first show that the finite-dimensional distributions converge and then that the family of approximating processes is tight. In particular, we have the following standard result, adapted from Billingsley [2, Thm. 7.5]. We define the function $w_{\delta,[0, T]}$ on $C[0, T]$ by

$$
w_{\delta,[0, T]}(U)=\sup _{t_{1}, t_{2} \in[0, T],\left|t_{2}-t_{1}\right|<\delta}\left|U\left(t_{2}\right)-U\left(t_{1}\right)\right| .
$$

Theorem 2.3 Let $U_{n}, n \geq 1, U$ be processes in $C[0, T]$ such that

(i) Finite-dimensional distributions converge: For all $t_{1}, \ldots, t_{d} \in[0, T]$ we have that

$$
\left.\left\{U_{n}\left(t_{j}\right)\right\}_{j=1 \ldots d} \Rightarrow\left\{U\left(t_{j}\right)\right)\right\}_{j=1 \ldots d}
$$

as $n \rightarrow \infty$. (That is, weak convergence in $\mathbb{R}^{d}$ ).

(ii) The processes satisfy a tightness criterion: For all $\epsilon>0$ and all $\eta>0$ there is a $\delta>0$ such that

$$
\mathbb{P}\left\{w_{\delta,[0, T]}\left(U_{n}\right) \geq \epsilon\right\} \leq \eta,
$$

for all $n$. Then $U_{n} \Rightarrow U$ in $C[0, T]$.

In Section 3 we establish the convergence of the finite-dimensional distributions and in Section 4 we establish the tightness criterion. We are able to prove the former result without the assumption of the velocity distribution being compactly supported. We conjecture that the main theorem holds without this assumption but could not obtain the tightness result without it.

Spitzer also divides his proof into proving convergence of finite-dimensional distributions and then establishing tightness. In his case, Spitzer accomplishes the former task with the use of characteristic functions (Fourier transforms of density functions). In our case, the fact that there is a finite number 
of particles allows us to avoid this and appeal directly to a version of the Central Limit Theorem for medians. Proving tightness for our family of trajectories cannot be done with Spitzer's techniques, since his arguments rely heavily on the processes having independent increments. Instead we observe that, if velocities are bounded, over short intervals of time the tracer particle undergoes motion that approximates in distribution that of the tracer in Spitzer's system. We are able to control this approximation sufficiently well to show that the trajectories in our system inherit the tightness of the trajectories in Spitzer's system.

\section{Convergence of Finite-Dimensional Distributions}

In this section we establish the convergence of the finite-dimensional distributions of the tracer particles' trajectories to those of the limiting Gaussian process. For any set of times $t_{1}, \ldots, t_{d} \geq 0$, the random vector $\left\{U_{n}\left(t_{j}\right)\right\}_{j=1 \ldots d}$ is the scaled median of the $n$ i.i.d. random vectors $\left\{y_{i}\left(t_{j}\right)\right\}_{j=1 \ldots d}$. We wish to show that this random vector converges weakly to the Gaussian random vector $\left\{U\left(t_{j}\right)\right\}_{j=1 \ldots d}$ as $n \rightarrow \infty$. This can be accomplished by a Central Limit Theorem (CLT) for vector medians of the type discussed in the book [18]. The general theorems proved in this reference do not cover our case, but the following proof borrows heavily from it.

In the proof we use the following characterization of weak convergence in $\mathbb{R}^{d}$. For a random vector $Y$ in $\mathbb{R}^{d}$, we define its distribution function $F_{Y}: \mathbb{R}^{d} \rightarrow \mathbb{R}$ by

$$
F_{Y}\left(x_{1}, \ldots, x_{d}\right):=\mathbb{P}\left\{Y(1) \leq x_{1}, \ldots, Y(d) \leq x_{d}\right\},
$$

where $Y(j)$ is the $j$ th component of $Y$. We say that $x \in \mathbb{R}^{d}$ is a continuity point of the random vector $Y$ if $F_{Y}$ is continuous at $x$. Random vectors $Y_{n}, n \geq 1$ converge weakly to $Y$ if and only if for all continuity points $x$ of $Y$,

$$
\mathbb{P}\left\{Y_{n}(j) \leq x_{j}, j=1, \ldots, d\right\} \rightarrow \mathbb{P}\left\{Y(j) \leq x_{j}, j=1, \ldots, d\right\},
$$

as $n \rightarrow \infty[2]$.

Lemma 3.1 Let the velocity density $f$ satisfy conditions (i) and (ii) of Assumptions 2.1. Then the finite-dimensional distributions of $U_{n}$ converge 
weakly to those of $U$. That is, for any $t_{1}, \ldots, t_{d} \in[0, \infty)$,

$$
\left\{U_{n}\left(t_{j}\right)\right\}_{j=1 \ldots d} \Rightarrow\left\{U\left(t_{j}\right)\right\}_{j=1 \ldots d},
$$

as $n \rightarrow \infty$.

Proof Let $x \in \mathbb{R}^{d}$ be a continuity point of $\left\{U\left(t_{j}\right)\right\}_{j=1 \ldots d}$ with components $x_{j}, j=1, \ldots, d$. We use the characterization of weak convergence in $\mathbb{R}^{d}$ given in (3.7). Consider the event of interest:

$$
\begin{aligned}
\left\{U_{n}\left(t_{j}\right) \leq x_{j}, j=1, \ldots, d\right\} & =\left\{n^{1 / 2} x_{\text {med }}\left(t_{j}\right) \leq x_{j}, j=1, \ldots, d\right\} \\
& =\left\{x_{\text {med }}\left(t_{j}\right) \leq n^{-1 / 2} x_{j}, j=1, \ldots, d\right\} .
\end{aligned}
$$

Recall that $x_{\text {med }}(t)$ is the median of $y_{i}(t), i=1, \ldots, n$. Using a standard identity for medians we have for each $j$

$$
\left\{x_{\text {med }}\left(t_{j}\right) \leq n^{-1 / 2} x_{j}\right\}=\left\{\sum_{i=1}^{n} \mathbf{1}_{y_{i}\left(t_{j}\right) \leq n^{-1 / 2} x_{j}} \geq n / 2\right\},
$$

where $\mathbf{1}_{A}$ is the indicator function of the event $A$. So

$$
\left\{U_{n}\left(t_{j}\right) \leq x_{j}\right\}=\left\{-\sum_{i=1}^{n} \mathbf{1}_{y_{i}\left(t_{j}\right) \leq n^{-1 / 2} x_{j}} \leq-n / 2\right\} .
$$

Let us manipulate the event on the right of the equation. We subtract the expectation of the left-hand-side of the inequality from both sides. Since

$$
\mathbb{E}\left(-\mathbf{1}_{y_{i}\left(t_{j}\right) \leq n^{-1 / 2} x_{j}}\right)=-\left(\frac{1+n^{-1 / 2} x_{j}}{2}\right)
$$

we obtain

$$
\begin{aligned}
& \left\{U_{n}\left(t_{j}\right) \leq x_{j}\right\} \\
& =\left\{\sum_{i=1}^{n}\left[\frac{1+n^{-1 / 2} x_{j}}{2}-\mathbf{1}_{y_{i}\left(t_{j}\right) \leq n^{-1 / 2} x_{j}}\right] \leq \sum_{i=1}^{n} \frac{n^{-1 / 2} x_{j}}{2}\right\} \\
& =\left\{n^{-1 / 2} \sum_{i=1}^{n}\left[1+n^{-1 / 2} x_{j}-2 \mathbf{1}_{y_{i}\left(t_{j}\right) \leq n^{-1 / 2} x_{j}}\right] \leq x_{j}\right\} .
\end{aligned}
$$


Let us define

$$
\nu_{n, i, j}:=1+n^{-1 / 2} x_{j}-2 \mathbf{1}_{y_{i}\left(t_{j}\right) \leq n^{-1 / 2} x_{j}}
$$

and

$$
N_{n, j}:=n^{-1 / 2} \sum_{i=1}^{n} \nu_{n, i, j}
$$

Then

$$
\begin{aligned}
\left\{U_{n}\left(t_{j}\right) \leq x_{j}, j=1, \ldots, d\right\} & =\left\{N_{n, j} \leq x_{j}, j=1, \ldots, d\right\} \\
& =\left\{n^{-1 / 2} \sum_{i=1}^{n} \nu_{n, i, j} \leq x_{j}, j=1, \ldots, d\right\}
\end{aligned}
$$

The event on the right is now in a form amenable to a multivariate central limit theorem. In the appendix we show that $\left\{N_{n, j}\right\}_{j=1 \ldots d}$ weakly converges to a Gaussian random vector with covariance matrix $\Sigma_{i j}=C\left(t_{i}-t_{j}\right)$. Therefore,

$$
\mathbb{P}\left\{N_{n, j} \leq x_{j}, j=1, \ldots, d\right\} \rightarrow \mathbb{P}\left\{U\left(t_{j}\right) \leq x_{j}, j=1, \ldots, d\right\}
$$

since $x$ is a continuity point of $\left\{U\left(t_{j}\right)\right\}_{j=1 \ldots d}$. This establishes the desired result.

\section{Tightness Criterion}

Now that we have proven that the finite-dimensional distributions of $U_{n}$ converge to those of $U$, it remains to show that the sequence of processes $U_{n}$ satisfies the tightness criterion of Theorem 2.3. We will do this by describing another construction of the processes $U_{n}$. First, we will provide a construction of the process in Spitzer's paper [20], $V_{n} \in C[0, \infty), n \geq 1$. Then we will apply a random transformation to $V_{n}$ to obtain another sequence of processes $\hat{U}_{n}$, defined on the same probability space, that also satisfies the tightness criterion. This sequence of processes will have the advantage that they are identical with $U_{n}$ on a certain time interval with high probability. This will allow us to establish the tightness criterion for $U_{n}$. Our approach here is similar to that used to prove tightness in another context in [5].

We now describe one possible construction of Spitzer's processes. For each odd $n \geq 1$ we place particles on the real line according to a uniform Poisson process with constant density $n / 2$. We add a tracer particle at the origin. 
We denote the particle positions with $\ldots z_{-2} \leq z_{-1} \leq z_{0}=0 \leq z_{1} \leq z_{2} \ldots$. We give the particles i.i.d. velocities $w_{j}$ from the density $f$. The motion of the particles is the same as for our system but without walls: instantaneous momentum- and energy-conserving collisions with free motion otherwise. At times $t \geq 0$ we multiply the position of the tracer particles by $n^{1 / 2}$ to get $V_{n}(t)$. A more formal definition of $V_{n}$ is

$$
V_{n}:=n^{1 / 2} \lim _{p \rightarrow \infty} \operatorname{med}_{i=-p \ldots p}\left(z_{i}+t w_{i}\right),
$$

which is shown in [12] to agree with the physical definition above. Spitzer's paper proves that $V_{n}$ weakly converges to Brownian motion (with some nonrandom constant scaling in time). The result of interest is that $V_{n}$ is a tight sequence of processes in $C[0, T]$ for all $T>0$ and that the second condition of Theorem 2.3 is satisfied by them [2]. Moreover, the set of velocity distributions that Spitzer obtains his result for contains the set of distributions that satisfy Assumptions 2.1.

We now make some comments about the initial inter-particle spacing in this system. Recall that for a uniform Poisson process on the real line, the inter-particle spacings are i.i.d. exponentially distributed random variables. Indeed, the addition of the tracer particle at 0 does not change this fact [20]. If we define $\xi_{i}=z_{i}-z_{i-1}$ for all $i$, the $\xi_{i}$ are i.i.d. exponentially distributed random variables with mean $2 / n$.

Each process $V_{n}$ is defined on a probability space of bi-infinite sequences $\left(\left\{z_{i}\right\},\left\{w_{i}\right\}\right)$. For each odd $n$ we shall define a new process $\hat{U}_{n}(t)$ on the same space. Let $m=(n-1) / 2$. We define the random variables

$$
\rho_{n}=\frac{z_{m+1}-z_{-m-1}}{2}, \quad \mu_{n}=\frac{z_{m+1}+z_{-m-1}}{2} .
$$

Note that

$$
\rho_{n}=\frac{1}{2} \sum_{i=-m}^{m+1} \xi_{i}, \quad \mathbb{E} \rho_{n}=\frac{n+1}{n} .
$$

We let

$$
\hat{z}_{i}=\left(z_{i}-\mu_{n}\right) / \rho_{n}
$$

for all $i$. We have performed an affine transformation to the positions of the particles so that $\hat{z}_{m+1}=1$ and $\hat{z}_{-m-1}=-1$. Now, with probability one, there are exactly $n$ particles in the open interval $(-1,1)$. We define $\hat{U}_{n}$ as we 
did $V_{n}$ before, but with the initial particle positions $\left\{\hat{z}_{i}\right\}$. So

$$
\hat{U}_{n}(t):=n^{1 / 2} \lim _{p \rightarrow \infty} \operatorname{med}_{i=-p \ldots p}\left(\hat{z}_{i}+t w_{i}\right) .
$$

Using (4.11) and (4.12) we can obtain an explicit relationship between the two sequences of processes. For each $n$,

$$
\begin{aligned}
\hat{U}_{n}(t) & =n^{1 / 2} \lim _{p \rightarrow \infty} \underset{i=-p \ldots p}{\operatorname{med}}\left(\left(z_{i}-\mu_{n}\right) / \rho_{n}+t w_{i}\right) \\
& =\rho_{n}^{-1} n^{1 / 2}\left[\lim _{p \rightarrow \infty} \operatorname{med}_{i=-p \ldots p}\left(z_{i}+\left(\rho_{n} t\right) w_{i}\right)-\mu_{n}\right] \\
& =\left[V_{n}\left(\rho_{n} t\right)-n^{1 / 2} \mu_{n}\right] / \rho_{n} .
\end{aligned}
$$

Using this relation we now establish that the family of processes satisfies the tightness criterion.

Lemma 4.1 The sequence of processes $\hat{U}_{n}(t), n \geq 1, n$ odd, satisfies the second condition of Theorem 2.3 in $C[0, T]$ for any $T>0$.

Proof Let us fix an interval $[0, T]$. Recall the definition of $w_{\delta,[0, T]}$ from (2.6). First note that

$$
\begin{aligned}
w_{\delta,[0, T]}\left(\hat{U}_{n}\right) & =\sup _{t_{1}, t_{2} \in[0, T],\left|t_{1}-t_{2}\right|<\delta}\left|\hat{U}_{n}\left(t_{2}\right)-\hat{U}_{n}\left(t_{1}\right)\right| \\
& =\sup _{t_{1}, t_{2} \in[0, T],\left|t_{1}-t_{2}\right|<\delta}\left|V_{n}\left(\rho_{n} t_{2}\right)-V_{n}\left(\rho_{n} t_{1}\right)\right| / \rho_{n} \\
& =\sup _{\hat{t}_{1}, \hat{t}_{2} \in\left[0, \rho_{n} T\right],\left|\hat{t}_{1}-\hat{t}_{2}\right|<\rho_{n} \delta}\left|V_{n}\left(\hat{t}_{2}\right)-V_{n}\left(\hat{t}_{1}\right)\right| / \rho_{n} \\
& =\rho_{n}^{-1} w_{\rho_{n} \delta,\left[0, \rho_{n} T\right]}\left(V_{n}\right),
\end{aligned}
$$

where we have used the substitution $\hat{t}=\rho_{n} t$. Now let us fix an $\epsilon>0$ and an $\nu>0$. We have that

$$
\begin{aligned}
\mathbb{P}\left\{w_{\delta,[0, T]}\left(\hat{U}_{n}\right) \geq \epsilon\right\} & =\mathbb{P}\left\{\rho_{n}^{-1} w_{\rho_{n} \delta,\left[0, \rho_{n} T\right]}\left(V_{n}\right) \geq \epsilon\right\} \\
& \leq \mathbb{P}\left\{\rho_{n} \notin[1 / 2,2]\right\}+\mathbb{P}\left\{w_{2 \delta,[0,2 T]}\left(V_{n}\right) \geq \epsilon / 2\right\}
\end{aligned}
$$

By (4.10) and the law of large numbers, $\rho_{n}$ converges almost surely to 1 . So we can choose $n_{1}$ such that $n \geq n_{1}$ implies that the first probability is less than $\nu / 2$. By the tightness of $V_{n}$ we can find an $n_{2}$ and a $\delta$ such that the 
second term is less than $\nu / 2$ for all $n \geq n_{2}$. Choosing $N=\max \left(n_{1}, n_{2}\right)$ gives the required result.

Our reason for introducing $\hat{U}_{n}$ is that the positions $\hat{z}_{-m}, \ldots, \hat{z}_{m}$ are now distributed identically to the order statistics of the positions $q_{1}, \ldots, q_{n}$ of our original system described in Section 2. To see this, note that we can write the $\hat{z}_{i}$ as

$$
\begin{aligned}
\hat{z}_{-m-1+j} & =\left(z_{-m-1+j}-\mu_{n}\right) / \rho_{n} \\
& =\left(z_{-m-1}+\left(\sum_{i=-m}^{-m-1+j} \xi_{i}\right)-\left(z_{-m-1}+z_{m+1}\right) / 2\right) / \rho_{n} \\
& =-1+2\left(\sum_{i=-m}^{-m-1+j} \xi_{i}\right) /\left(\sum_{i=-m}^{m+1} \xi_{i}\right) .
\end{aligned}
$$

Since the $\xi_{i}$ are i.i.d. exponential random variables, the ratio of the sums of the $\xi_{i}$ in the above expression for $j=1, \ldots, n$ are distributed as the order statistics of $n$ i.i.d. uniform random variables on [0,1] [6, p. 148]. So $\hat{z}_{-m-1-j}$ for $j=1, \ldots, n$ are distributed as the order statistics of $n$ i.i.d. uniform random variables on $[-1,1]$.

This gives us a way of defining our original process $U_{n}$ on the same space as $V_{n}$ and $\hat{U}_{n}$. Before we defined $U_{n}(t)=\operatorname{med}_{i=1 \ldots n} G\left(q_{i}+p_{i}\right)$ where $q_{i}$ were i.i.d. uniform random variables on $[-1,1]$. However, since the $p_{i}$ are distributed independently of the $q_{i}$, this is identical in distribution to defining $U_{n}$ as $\operatorname{med}_{i=1 \ldots n} G\left(q_{[i]}+p_{i}\right)$ where $q_{[j]}$ is the $j$ th order statistic of the $q_{i}$. Therefore, we can define $U_{n}$ by

$$
U_{n}(t)=\underset{i=-m \ldots m}{\operatorname{med}} G\left(\hat{z}_{i}+w_{i} t\right) .
$$

Physically, this has the following interpretation. To generate the random trajectory $U_{n}$ we set up the particles as we did for $\hat{U}_{n}$. But now we remove all but the $n$ particles with positions $\hat{z}_{-m}, \ldots, \hat{z}_{m}$ (which are all in $[-1,1]$ ) and add walls at -1 and 1 . We then let the particles move and interact with each other and with the walls as before. The trajectory of the tracer particle multiplied by $n^{1 / 2}$ gives us $U_{n}$. The trajectory of the particles will be different for $U_{n}$ and $\hat{U}_{n}$. However, since particle speed is bounded by $V_{\max }$ according to Assumption 2.1(iii), there will be a period of time during which, with high probability, the trajectory of the tracer will not be affected. This 
is the content of the following theorem.

Lemma 4.2 The probability that $\hat{U}_{n}$ and $U_{n}$ are identical on the time-interval $\left[0,1 / 4 V_{\text {max }}\right]$ goes to 1 as $n \rightarrow \infty$.

Proof Because the maximum particle speed is less than $V_{\max }$ and the maximum relative particle speed is less than $2 V_{\max }$, the tracer particle cannot be affected on the time interval $\left[0,1 / 4 V_{\max }\right]$ by anything that is further than distance $1 / 2$ away from it. In particular, if the particle is further than distance $1 / 2$ from the walls at time 0 it will not be able to distinguish the difference between the set-ups for $\hat{U}_{n}$ and $U_{n}$ for that interval of time, and the two processes will be identical. Now,

$$
\begin{aligned}
\mathbb{P}\left\{\hat{z}_{0}>1 / 2\right\} & =\mathbb{P}\left\{\sum_{i=1 \ldots n} q_{i}>1 / 2\right\} \\
& =\mathbb{P}\left\{\sum_{i=1 \ldots n} \mathbf{1}_{q_{i}>1 / 2}>n / 2\right\} \\
& =\mathbb{P}\left\{n^{-1} \sum_{i=1 \ldots n} \mathbf{1}_{q_{i}>1 / 2}>1 / 2\right\} .
\end{aligned}
$$

Since the $\mathbb{E}\left\{\mathbf{1}_{q_{i}>1 / 2}\right\}=\mathbb{P}\left\{q_{i}>1 / 2\right\}=1 / 4$ the probability in question converges to 0 as $n \rightarrow \infty$ by the law of large numbers.

With these two lemmas we can establish the tightness result.

Theorem 4.3 The family of processes $U_{n}(t), n \geq 1$ satisfies the second criterion of Theorem 2.3 in $C[0, T]$ for any $T>0$.

Proof Fix a $T>0$. Let $\epsilon, \nu>0$ be given. Let $M$ be an integer such that $M / 4 V_{\max } \geq T$. For any $\delta>0$

$$
\begin{aligned}
w_{\delta,[0, T]}\left(U_{n}\right) & \leq w_{\delta,\left[0, M / 4 V_{\max }\right]}\left(U_{n}\right) \\
& \leq 2 \max _{i=1 \ldots M} w_{\delta,\left[(i-1) / 4 V_{\max }, i / 4 V_{\max }\right]}\left(U_{n}\right) .
\end{aligned}
$$

Since $U_{n}$ is a stationary process, $w_{\delta,\left[(i-1) / 4 V_{\max }, i V_{\max } / 2\right]}\left(U_{n}\right)$ has the same distribution regardless of $i$. So

$$
\begin{aligned}
\mathbb{P}\left\{w_{\delta,[0, T]}\left(U_{n}\right) \geq \epsilon\right\} & \leq \sum_{i=1 \ldots M} \mathbb{P}\left\{w_{\delta,\left[(i-1) / 4 V_{\max }, i V_{\max } / 2\right]}\left(U_{n}\right) \geq \epsilon / 2 M\right\} \\
& =M \mathbb{P}\left\{w_{\delta,\left[0,1 / 4 V_{\max }\right]}\left(U_{n}\right) \geq \epsilon / 2 M\right\}
\end{aligned}
$$


This last probability is less than or equal to

$$
\mathbb{P}\left\{U_{n} \not \equiv \hat{U}_{n} \text { on }\left[0,1 / 4 V_{\text {max }}\right]\right\}+\mathbb{P}\left\{w_{\delta,\left[0,1 / 4 V_{\text {max }}\right]}\left(\hat{U}_{n}\right) \geq \epsilon / 2 M\right\} .
$$

The first term can be made less than $\nu / 2$ by choosing $n \geq n_{1}$ for some $n_{1}$, by the previous lemma. Since $\hat{U}_{n}$ is tight, we can choose a $\delta>0$ and an $n_{2}$ such that the second term is less than $\nu / 2$ for $n \geq n_{2}$. Using this $\delta$ and $N=\max \left(n_{1}, n_{2}\right)$ gives us the result we need for $U_{n}$.

\section{Approximation with Soft Collisions}

We have now shown that a particular sequence of deterministic processes with random initial conditions has a component $U_{n}$, defined by $(2.3)$, that converges to a mean-zero Gaussian process $U$ specified by (2.5). As mentioned earlier, since these deterministic processes are not described purely by ODEs we cannot test ODE solvers on them in a straightforward fashion. So we shall now describe an approximation of these systems by systems of ODEs. We will then provide numerical evidence that the trajectories of the tracer particles of these ODE systems also converges to the stochastic process $U$ in a particular limit.

As before, for each odd positive integer $n$, we place $n$ particles independently on the interval $\left[-n^{1 / 2}, n^{1 / 2}\right]$ according to a uniform distribution. Each particle is independently given a velocity $n^{1 / 2} p$ where $p$ is selected from the distribution with density $f$, as before. The two choices of $f$ we will consider are (i) standard normal distribution, (ii) uniform on $[-1,1]$. Though our main convergence theorem does not apply in case (i), the convergence of the finite-dimensional distributions does and that is what we shall consider in our experiments. We denote the position and velocity of particle $i$ at time $t$ by $q_{i}(t)$ and $p_{i}(t)$ respectively, with $q_{i+1}(0)>q_{i}(0)$. In contrast to the previous case, we describe the motion of the particles through a set of ODEs. The differential equations describing the positions $q_{i}$ and the momenta $p_{i}$ are

$$
\dot{q}_{i}(t)=p_{i}(t), \quad i=1, \ldots, n,
$$


and

$$
\begin{aligned}
\dot{p}_{1}(t) & =-k^{2}\left(q_{1}(t)-q_{2}(t)\right)_{+}+k^{2}\left(-n^{1 / 2}-q_{1}(t)\right)_{+}, \\
\dot{p}_{i}(t) & =-k^{2}\left(q_{i}(t)-q_{i+1}(t)\right)_{+}+k^{2}\left(q_{i-1}(t)-q_{i}(t)\right)_{+}, \quad i=2, \ldots, n-1, \\
\dot{p}_{n}(t) & =-k^{2}\left(q_{n}(t)-n^{1 / 2}\right)_{+}+k^{2}\left(q_{n-1}(t)-q_{n}(t)\right)_{+},
\end{aligned}
$$

where $(x)_{+}=\max (0, x)$ for $x \in \mathbb{R}$. This system can be viewed as a Hamiltonian system with Hamiltonian

$$
H(q, p)=V(q)+\frac{1}{2} \sum_{i=1}^{n} p_{i}^{2}
$$

where

$$
V(q)=\frac{1}{2} k^{2}\left(-n^{1 / 2}-q_{1}\right)_{+}^{2}+\frac{1}{2} \sum_{i=2}^{n-1} k^{2}\left(q_{i-1}-q_{i}\right)_{+}^{2}+\frac{1}{2} k^{2}\left(q_{n}-n^{1 / 2}\right)_{+}^{2} .
$$

The tracer is chosen as the particle with the median position at time zero.

The flow of this system of ODEs is very similar to that of the original process. However, when two particles meet each other, rather than instantaneously exchanging velocities, they are allowed to overlap. While overlapping, they apply a repelling force to each other that is proportional to the amount that they have overlapped. Thus, while the particles are in contact, they go through simple harmonic motion. After half a period of this motion, they cease to overlap and have velocities pointing away from each other. Since momentum and energy are conserved, the net effect is that their velocities are exchanged, as in the hard collision case. However, their positions are displaced relative to where they would be after a hard collision. This only describes a two-particle collision: collisions with three or more particles occur with non-zero probability and are more complicated.

In the ODEs above, $k$ is a constant denoting the stiffness of the repulsion between the particles, and between the particles and the walls. For $k=0$ the particles do not interact at all; as $k \rightarrow \infty$ we expect the trajectories of the system to converge to those of the original system with the same initial data. (See, for example, [17] or [22].)

In order to have a sequence of systems of ODEs that converges to the same stochastic process as the system we considered previously, we allow $k$ to go to infinity as $n$ does. Here, we make the choice $k=\gamma n^{2}$, for some 
positive $\gamma$. In this case the duration of a collision between two particles is on the order of $n^{-2}$. The average length of time between collisions for a single particle is on the order of $n^{-1}$. So, as we increase $n$, the collision durations become shorter with respect to the length of time between collisions, and in the limit of $n \rightarrow \infty$ we expect the system to be a close approximation to the system with instantaneous collisions. We conjecture that the ODE system, with this choice of $k$, has the same limiting properties as those we proved for the instantaneous collision system: the trajectory of the tracer particles weakly converges to $U$. We believe this holds true for all positive $\gamma$, though in the following we fix $\gamma=1$. We now provide some numerical evidence to support this conjecture in this case.

As before, $C$ is the covariance of the limiting process $U$ which depends on $f$. Define $C_{n}$ to be the covariance of our original hard particle process with $n$ particles described in Section 2. Our main theorem in Section 3 shows that $C_{n}(t)$ converges to $C(t)$ for all $t$ for both choices of $f$ above. We denote by $C_{n, k}$ the covariance function for the process described by the ODEs with $n$ particles and a stiffness parameter $k$. A consequence of the softened system converging weakly to $U$ as $n \rightarrow \infty$ with $k=n^{2}$ would be the convergence of $C_{n, k}$ to $C$. For a given $n, k$ the natural way to obtain an accurate approximation to $C_{n, k}$ would be to numerically simulate the system with a time step so small that trajectories are accurately computed over the time interval of interest. We would expect that steps of size $\Delta t=\mathrm{o}(1 / k)=\mathrm{o}\left(1 / n^{2}\right)$ would be required for this. Unfortunately, this is impractical for larger values of $n$. Instead, let us define $C_{n, k, \Delta t}$ to be the autocovariance function for the numerical approximation to the system with $n$ particles, stiffness parameter $k$, and integration steplength $\Delta t$. We will compute $C_{n, k, \Delta t}$ for diminishing values of $\Delta t$ until we observe convergence. This converged value we will take as our estimate to $C_{n, k}$. In this section we will use exclusively the symplectic Euler method, given by the formulae:

$$
\begin{aligned}
& q^{n+1}=q^{n}+\partial H / \partial p\left(q^{n}, p^{n}\right) \\
& p^{n+1}=p^{n}-\partial H / \partial q\left(q^{n+1}, p^{n+1}\right)
\end{aligned}
$$

where $H$ is given by (5.13) and (5.14). This is explicit in our case since $\partial H / \partial q$ does not depend on $p$.

We demonstrate this procedure for the Gaussian velocity distribution in Figure 5.1. For $n=3,15,75$ with $k=n^{2}$, we show $C_{n, k, \Delta t}-C_{n}$ for a range of $\Delta t$. We subtract the function $C_{n}$ so that it is easier to see the differences 

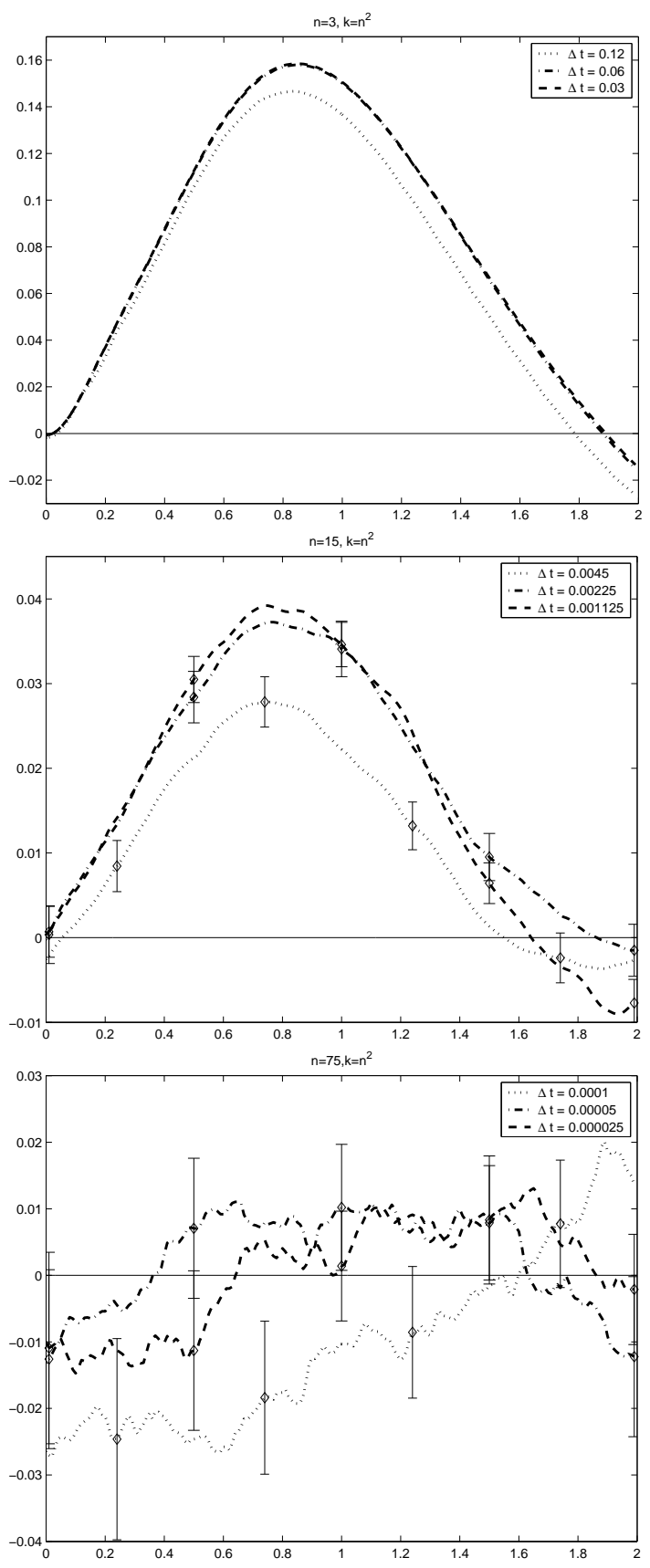

Figure 5.1: $C_{n, k, \Delta t}-C_{n}$ for three choices of $n, k$ for a range of $\Delta t$ with the Gaussian initial velocity distribution. On the first graph the standard deviation of the error is approximately the thickness of the lines; on the second and third representative error ${ }_{1}$ gars are shown. 
between $C_{n, k, \Delta t}$ for different $\Delta t$ on one graph. (Note that the scale on the $y$ axis differs in each plot.) For each $n, C_{n, k, \Delta t}$ appears to converge as $\Delta t \rightarrow 0$ within the accuracy provided by our statistics. Moreover, convergence is attained with a stepsize of $\Delta t>1 / 2 k$. Similar results were found for the uniform velocity distribution as we show in Figure 5.2. Accordingly, we take results computed with symplectic Euler with the stepsize $\Delta t=1 / 2 k$ to be accurate estimates of $C_{n, k}$ for both velocity distributions.

To show that $C_{n, k}$ converges to $C$ with $k=n^{2}$, we take these estimated values and plot the difference $C-C_{n, k, \Delta t}, \Delta t=1 / 2 k$, for varying $n$. Figure 5.3 shows that the difference appears to converge to zero for both velocity distributions, though this is less clear in the case of the uniform velocity distribution. Of course, convergence of the covariance function does not guarantee convergence of the process, but the evidence partly confirms our hypothesis.

These calculations suggest two conclusions for the velocity distributions considered. Firstly, the system of ODEs above, with scaling $k=n^{2}$, has the same limiting statistical property for its tracer particle as the system with instantaneous collisions. Secondly, when the solution of the system of ODEs is numerically approximated by the symplectic Euler method with stepsize $\Delta t=1 / 2 k$, this stochastic limit is also recovered. Of course, another possible interpretation of the data would be that the system of ODEs does not capture the same stochastic limit, though it does when approximated by the symplectic Euler method over a range of stepsizes. We believe this latter possibility is quite unlikely.

\section{Numerical Approximation}

In the previous section we presented a sequence of particle systems described by ODEs and parametrized by $n$, the number of particles, and $k$ the stiffness of the collisions. If we let $k=n^{2}$, it appears that the trajectory of the tracer particle weakly converges to that of the stochastic process $U$ as $n$ goes to infinity. Moreover, when the system was integrated with the symplectic Euler method with a large stepsize $(\Delta t=1 / 2 k)$, the same stochastic limit was obtained. In this section we will examine how other methods perform in comparison.

For any given numerical integrator, as we increase $n$ (and thus $k$ ), it is necessary for $\Delta t$ to be reduced in order to capture any features of the 

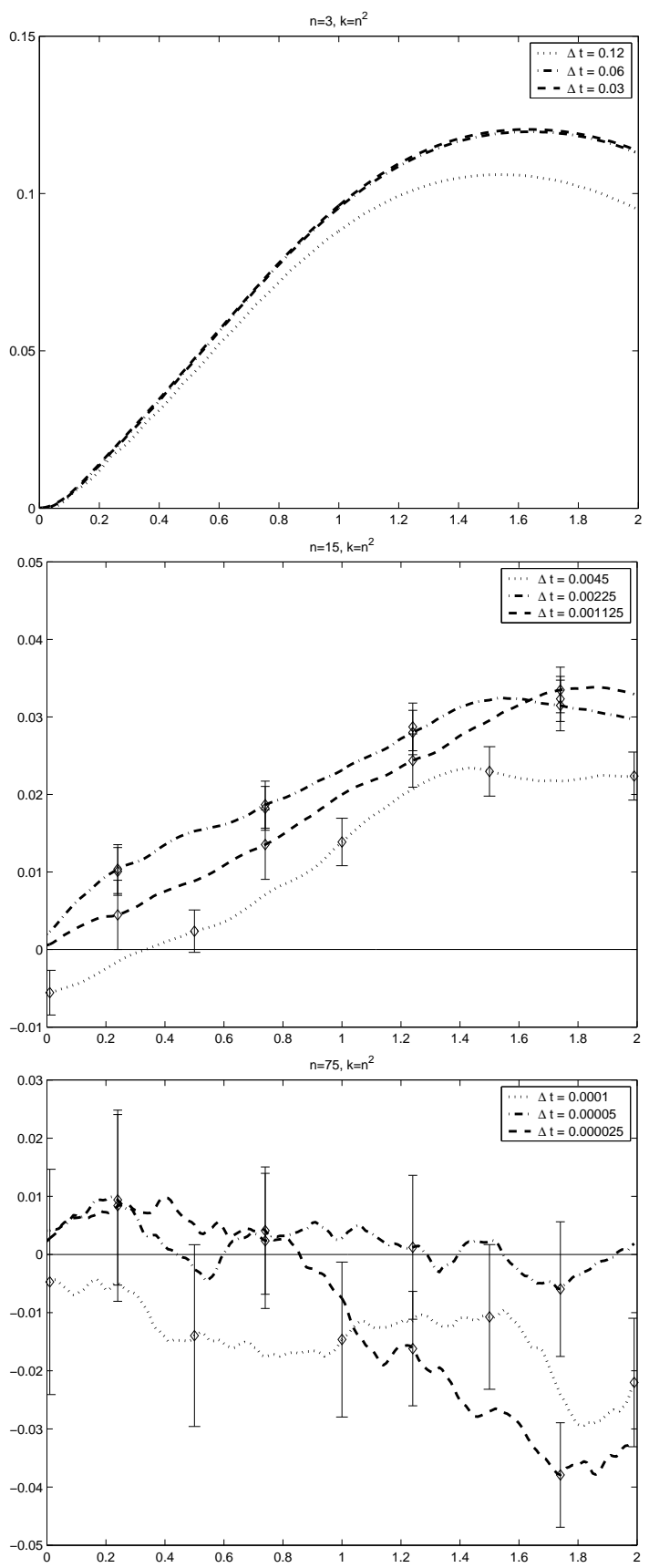

Figure 5.2: $C_{n, k, \Delta t}-C_{n}$ for three choices of $n, k$ for a range of $\Delta t$ with the uniform initial velocity distribution. On the first graph the standard deviation of the error is approximately the thickness of the lines; on the second and third representative erroretpars are shown. 

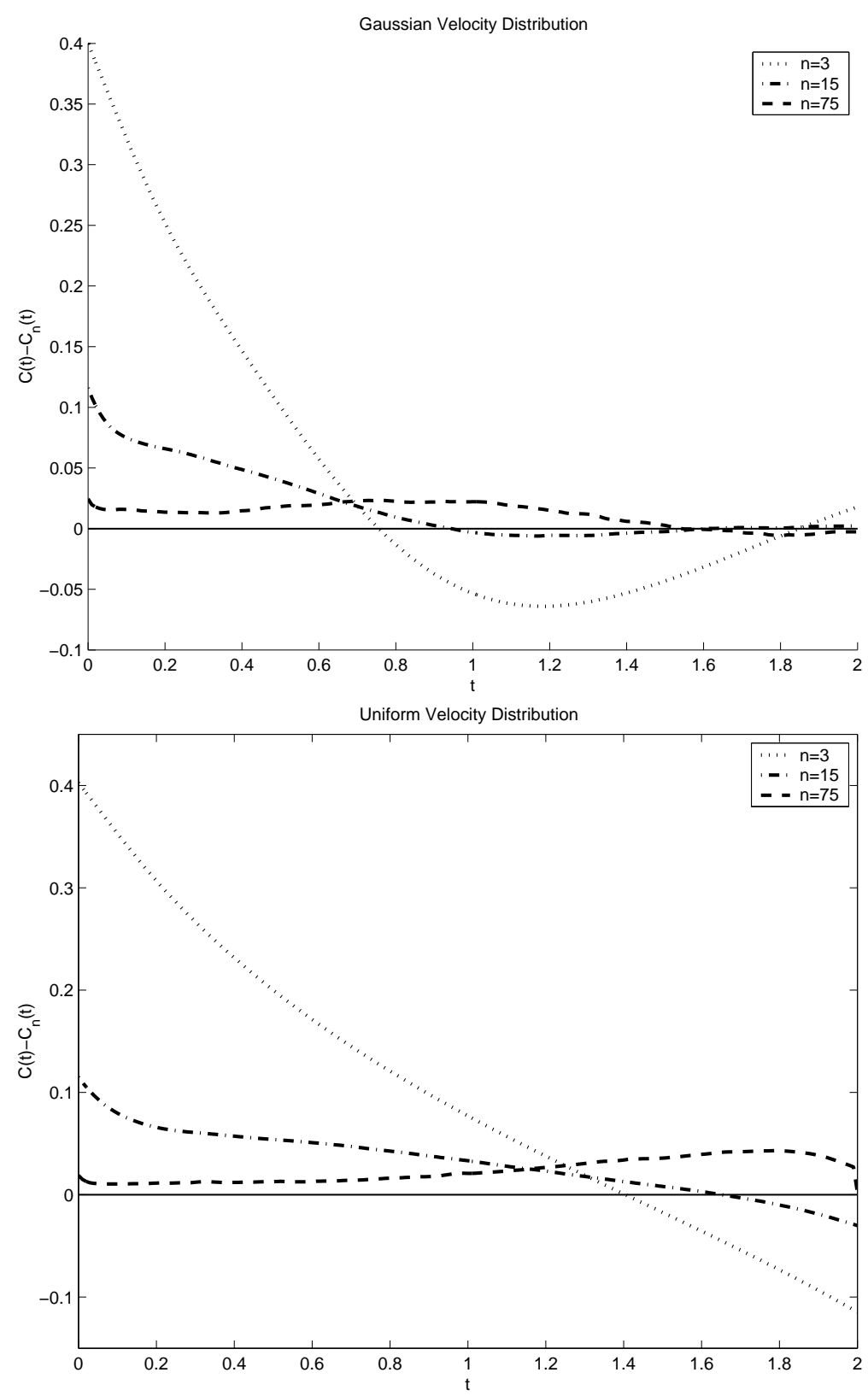

Figure 5.3: $C(t)-C_{n, k, \Delta t}(t)$ for $t \in[0,2]$ with $k=n^{2}, \Delta t=1 / 2 k$ for both velocity distributions considered. The standard deviation of the error on the curves never exceeds 0.004 . 
system. Since the duration of time two particles spend colliding is of order $1 / k$, to have an integrator take at least some steps during each collision as $n \rightarrow \infty$ it is necessary that $\Delta t=\mathcal{O}(1 / k)$ or smaller. We have already seen that symplectic Euler captures the limiting statistics with this scaling. For a method to be competitive with symplectic Euler near the stochastic limit, it must have $\Delta t=\mathcal{O}(1 / k)$ as well. (Indeed, we expect that if $\Delta t$ goes like $1 / k^{d}$ for $d>1$ then all convergent methods will capture statistics well on finite time intervals in the limit.) Accordingly, we shall test all methods with steplength $\Delta t=\gamma / k$ for some $\gamma>0$.

In Subsection 6.1 we will describe the five numerical methods we will consider. In Subsection 6.2 we will examine how each method performs computing individual tracjectories of the system for three values of $n$. We conclude in Subsection 6.3 by examining how the symplectic Euler method and its projected version fare at capturing the limiting statistical behaviour of the system.

\subsection{Numerical Methods}

We consider five numerical methods all used with a constant stepsize. The first is the symplectic Euler method as given in (5.15). The second and third are the standard methods backward Euler and forward Euler. The fourth is a fourth-order Runge-Kutta method [10], with Butcher tableau

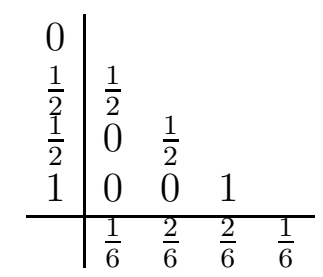

which we denote by RK4. Lastly, we consider a step-and-project method which we shall call projected symplectic Euler. This energy-conserving method requires some explanation.

One property of the symplectic Euler method which may be viewed as a drawback for molecular dynamics simulations is that it does not conserve the energy of the system exactly. One way to remedy this is to use a stepand-project method; see [9, IV.4]. A step-and-project method is a modified version of a standard time-stepping algorithm wherein the solution is forced to have the correct energy after each step. This is done by first taking a step 
with a standard method - which will lead to a value with possibly incorrect energy - and then projecting this value onto the manifold of states with the correct energy.

Typically, step-and-project methods for Hamiltonian systems are implemented as follows. Suppose we are numerically integrating a Hamiltonian system of ODEs on the space of points $x=(q, p) \in \mathbb{R}^{2 n}$ with fixed energy $H(x)=H_{0}$. From a state $x_{n}=\left(q_{n}, p_{n}\right)$ with the correct energy a standard time-stepper is used to obtain the state $x_{n+1}^{*}$. The gradient of the Hamiltonian is computed at this state: $s=\nabla H\left(x_{n+1}^{*}\right)$. Then an $\alpha \in \mathbb{R}$ is computed so that $H\left(x_{n+1}^{*}+\alpha s\right)=H_{0}$. Then we set $x_{n+1}=x_{n+1}^{*}+\alpha s$. (In our case an $\alpha$ was determined so that $\left|H\left(x_{n+1}\right)-H_{0}\right|<10^{-5}$.)

In our implementation there are two aspects of this scheme that we modify. Firstly, the we use a different projection direction $s$. The usual choice,

$$
s=\nabla H(q, p)=[\nabla V(q) p]^{T},
$$

does not scale well with increasing $k$. Instead we use

$$
s=\left[\begin{array}{ll}
\nabla V(q) & k^{2} p
\end{array}\right]^{T} .
$$

Secondly, for our system it is not always possible to find an $\alpha$ that solves the above nonlinear equation accurately enough. In these rare cases we find an $\alpha$ that locally minimizes the residual of the equation, and then proceed with the projection to $H(x)=H_{0}$ as usual on subsequent steps. In the following experiments, this occurs only for the $n=3$ case, and in less than $0.25 \%$ of the trials.

\subsection{Single Trajectories}

Before we consider these methods' ability to reproduce the stochastic limit, we will first examine the performance of each method on a single trajectory of the model system with $n=3,15,75$ and $k=n^{2}$, as in the previous section. In each case we plot the position of each particle versus time on the time interval $[0,4]$. The tracer particle is indicated by the darker, thicker line. Initial velocities were generated from the Gaussian distribution.

In Figure 6.1 we show trajectories computed with the symplectic Euler method with a step size of $\Delta t=1 / k$. This steplength is close to the largest that can be used without producing trajectories that quickly blow up due to 

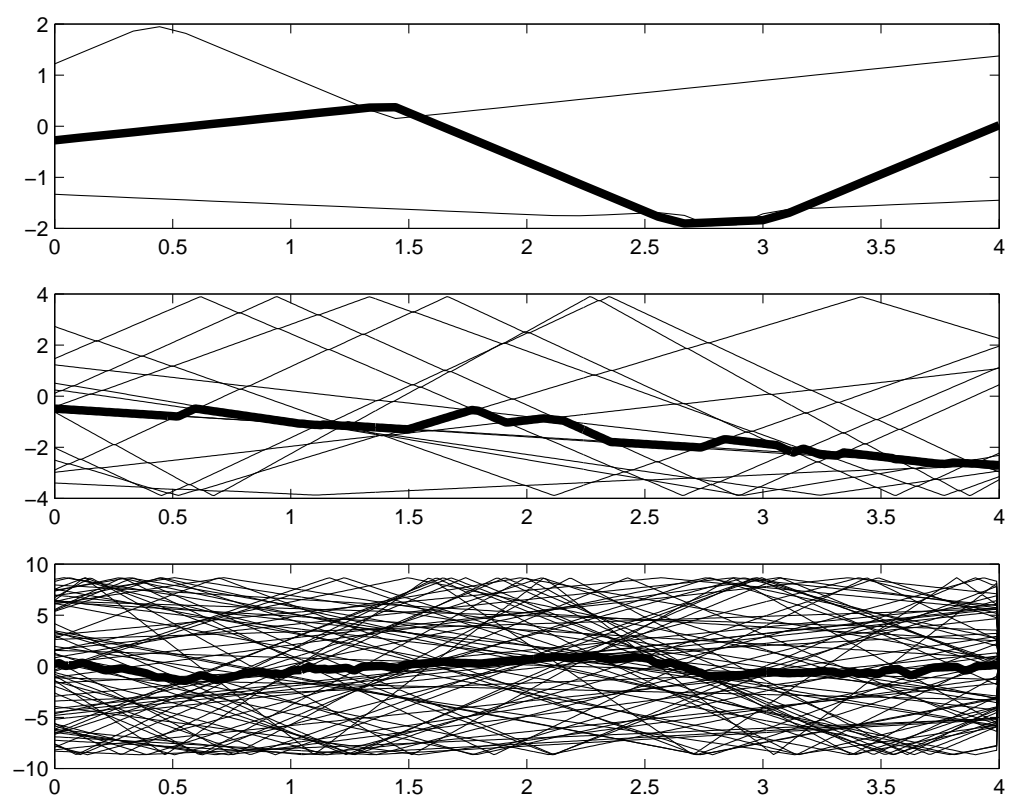

Figure 6.1: Trajectories computed with the symplectic Euler method with $\Delta t=1 / k$. Plots are of particle position versus time for $n=3,15,75, k=n^{2}$.

an increase in energy. This produces reasonable trajectories. In particular, we can see by looking where two particles collide that momentum is conserved (a property of all partitioned Runge-Kutta methods) and the energy of the two particles does not stray too far from what is expected.

Figure 6.2 shows trajectories computed with the backward Euler method with the same step length $\Delta t=1 / k$. This method has the shortcoming that particles tend to lose energy when they collide. This can already be observed for $n=3$ (by comparison with the figure above) but worsens as $n$ increases. For $n=75$ the particles quickly have very little energy and stick to the walls when they collide there. This could be improved by decreasing the step length, but backward Euler is already much more expensive than symplectic Euler, and could only be practical if a much longer timestep were possible. Moreover, we believe that with the scaling $\Delta t=\gamma k$, trajectories will become progressively worse with increasing $n$ for any $\gamma>0$.

In Figure 6.3 we show trajectories computed with forward Euler with $\Delta t=0.0039 / k$. Forward Euler has a strong tendency to add energy to the system at every collision. To obtain comparable performance with symplectic Euler for small $n$ it is necessary to take a vastly smaller step. Even with this 

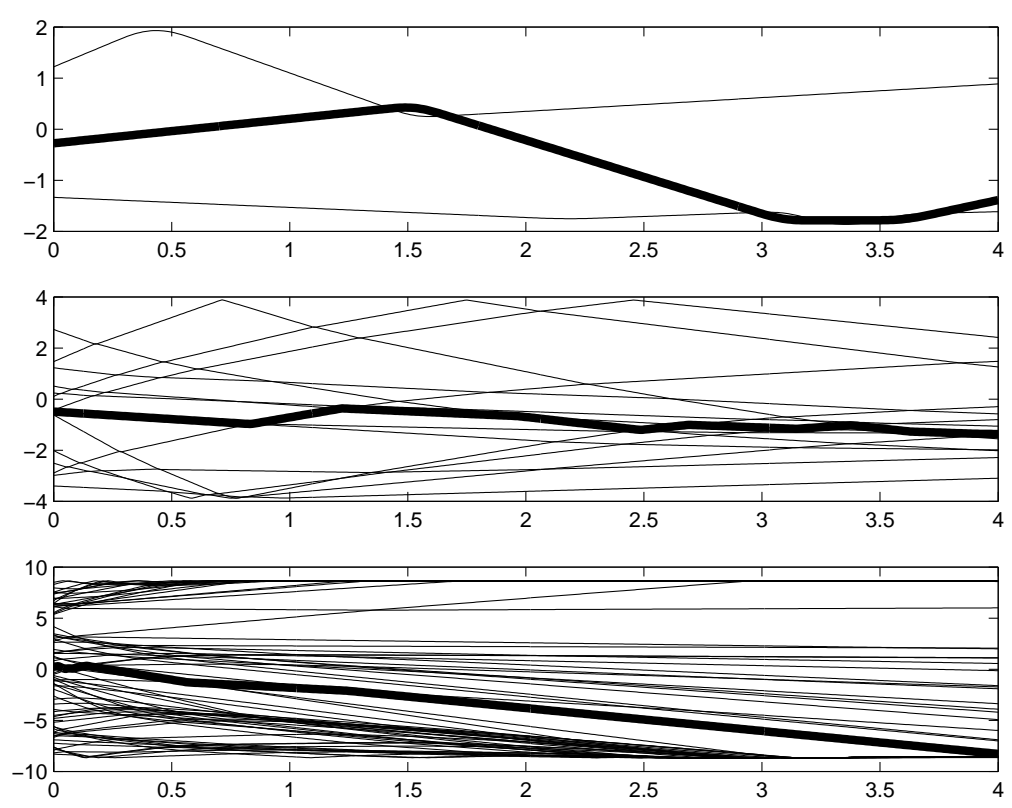

Figure 6.2: Trajectories computed with the backward Euler method with $\Delta t=1 / k$. Plots are of particle position versus time for $n=3,15,75, k=n^{2}$.

smaller step, for large $n$ the energy of the solution computed with forward Euler grows rapidly in time. We conjecture that if $\Delta t=\gamma / \Delta t$, for some $\gamma>0$, then the trajectory of the tracer particle will diverge as a stochastic process as $n \rightarrow \infty$.

Figure 6.4 shows trajectories computed with RK4. This method is, of course, more accurate than forward Euler for sufficiently small $\Delta t$. However, as $n$ increases the method also shows increasing instability in energy, and performs even worse than forward Euler if run with the same steplength. With a steplength of $\Delta t=0.00028 / k$, when $n=75$, overflow occurs near $t=3.5$ due to increasing energy.

Figure 6.5 shows trajectories computed with the projected symplectic Euler method with $\Delta t=1 / k$. In this case it is difficult to see any major difference between the this computation and that with the regular symplectic Euler method. However, since the projected symplectic Euler method is more costly, the method would not be practical unless a longer step-length could be used than with symplectic Euler.

Accordingly, Figure 6.6 shows trajectories computed with the same method with $\Delta t=1.25 / k$. However, this slight increase in $\Delta t$ has drastic effects. 

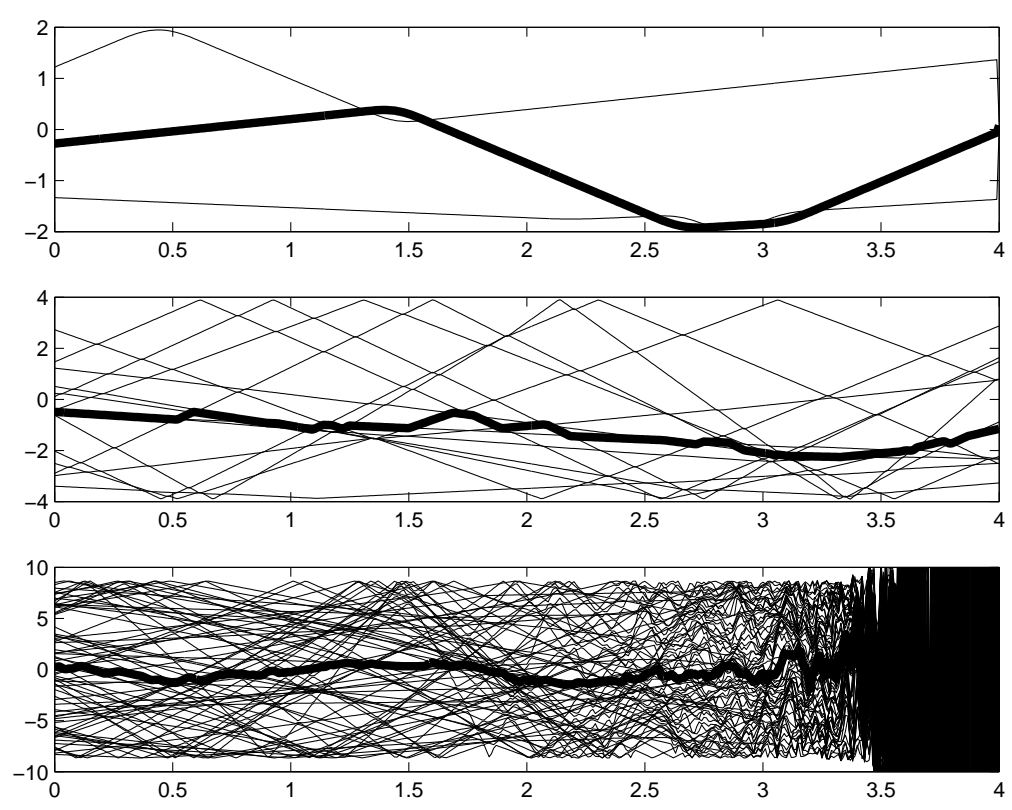

Figure 6.3: Trajectories computed with the forward Euler method with $\Delta t=$ $0.0039 / k$. Plots are of particle position versus time for $n=3,15,75, k=n^{2}$.
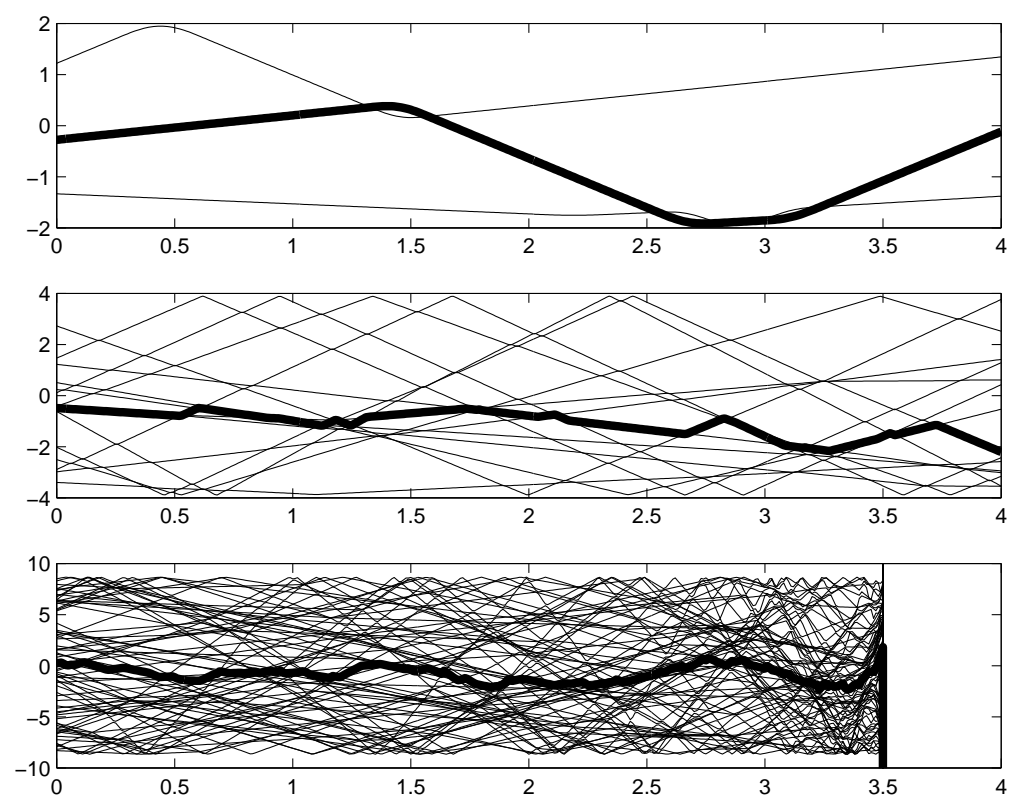

Figure 6.4: Trajectories computed with RK4 with $\Delta t=0.00028 / k$. Plots are of particle position versus time for $n=3,15,75, k=n^{2}$. 

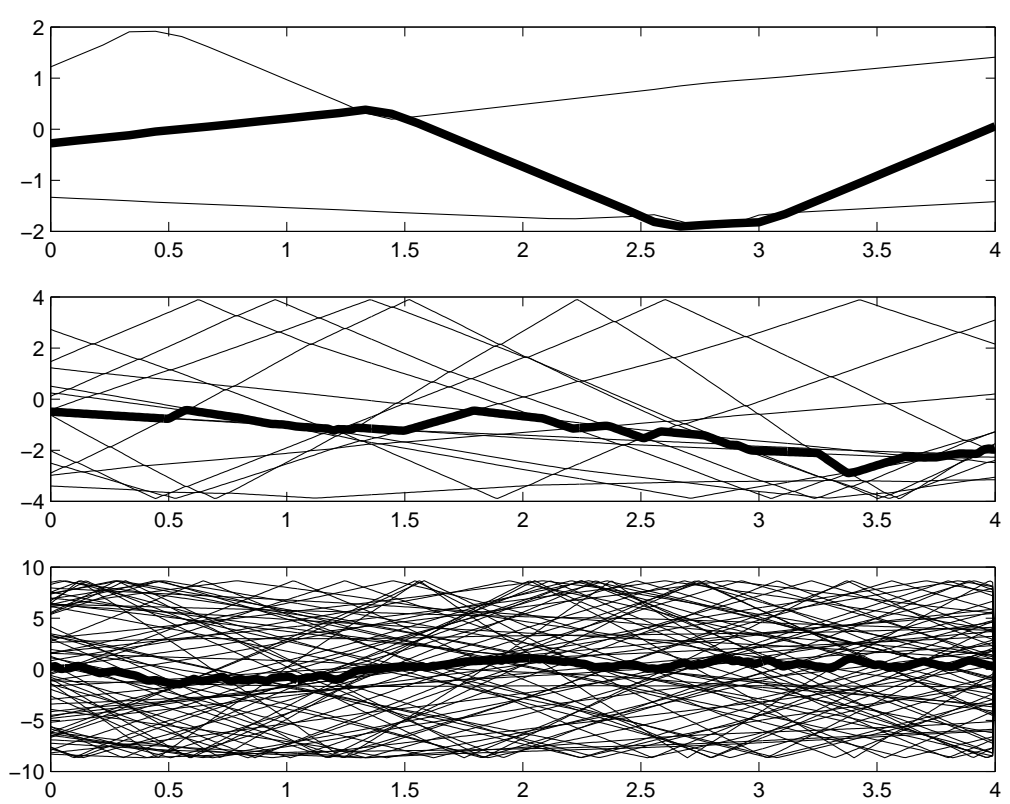

Figure 6.5: Trajectories computed with the projected symplectic Euler method with $\Delta t=1 / k$. Plots are of particle position versus time for $n=3,15,75, k=n^{2}$.

Even with $n=3$ we can see that the top particle's trajectory is being affected by the collisions of the other two particles at the other end of the interval. This unphysical behaviour is due to the energy projection. As $n$ increases, this becomes worse: when $n=75$ the net results of this non-local behaviour is that only a few particles have the vast majority of the energy in the system at any time.

\subsection{Limiting Statistics}

Clearly, neither the forward Euler, backward Euler, nor RK4 methods will capture long term statistics properly. Accordingly, for the our experimental investigation of the preservation of statistical limits, we only consider the symplectic Euler method and its projected version. In the following we will use the Gaussian distribution for the initial velocities.

Figure 6.7 shows $C-C_{n, k, \Delta t}$, with $C_{n, k, \Delta t}$ computed by the symplectic Euler method with $\Delta t=1 / k$, where $k=n^{2}$ and $n=3,15,75$. The covariance function appears to converge to the limiting covariance function $C$ with this 

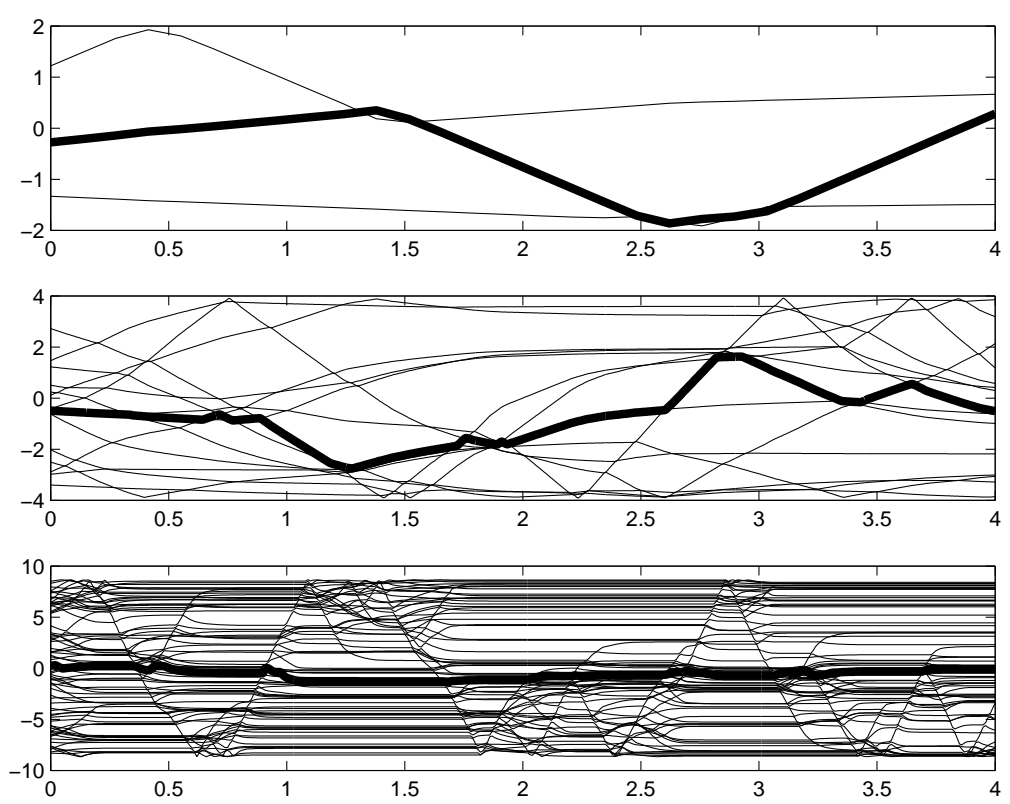

Figure 6.6: Trajectories computed with the projected symplectic Euler method with $\Delta t=1.25 / k$. Plots are of particle position versus time for $n=3,15,75, k=n^{2}$.

scaling. This provides an example of the phenomenon which we hoped to explore. Since with the $\Delta t \sim 1 / k$ scaling we are not resolving fine details of the collisions, we are not computing trajectories accurately in this limit.

We perform the same numerical experiment with the projected symplectic Euler method. Figure 6.8 shows $C-C_{n, k, \Delta t}$ computed by this method. As before $\Delta t=1 / k, k=n^{2}$, and $n=3,15,75$. Comparison with Figure 6.7 shows that for $n=3,15$ the method computes similar covariance functions to those of the non-projected method. However, for $n=75$ the covariance function appears different. It is difficult to ascertain from this plot whether convergence will occur as $n \rightarrow \infty$ with this step length $\Delta t=1 / k$. In either case, the covariance function is not converging to $C$ as rapidly as the regular symplectic Euler method converges, thus we know that the projection has some effect on statistics.

We repeat the experiment with the projected method with a slightly larger timestep: $\Delta t=1.251 / k$. The results shown are significantly worse than for the previous steplength. The computed covariance function is clearly not converging to $C$ as $n \rightarrow \infty$. These observations correspond with what we 


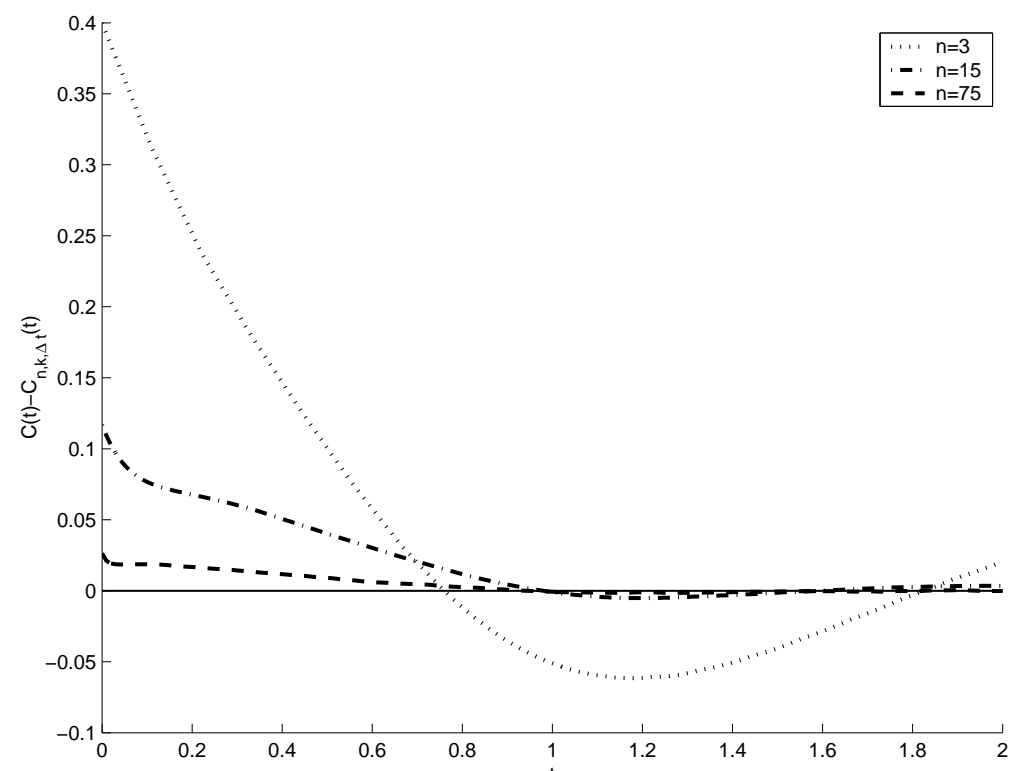

Figure 6.7: Symplectic Euler method. $C(t)-C_{n, k, \Delta t}(t)$ for $t \in[0,2]$, with $\Delta t=1 / k$, and $k=n^{2}$. The standard deviation of the error on the curves never exceeds 0.002 .

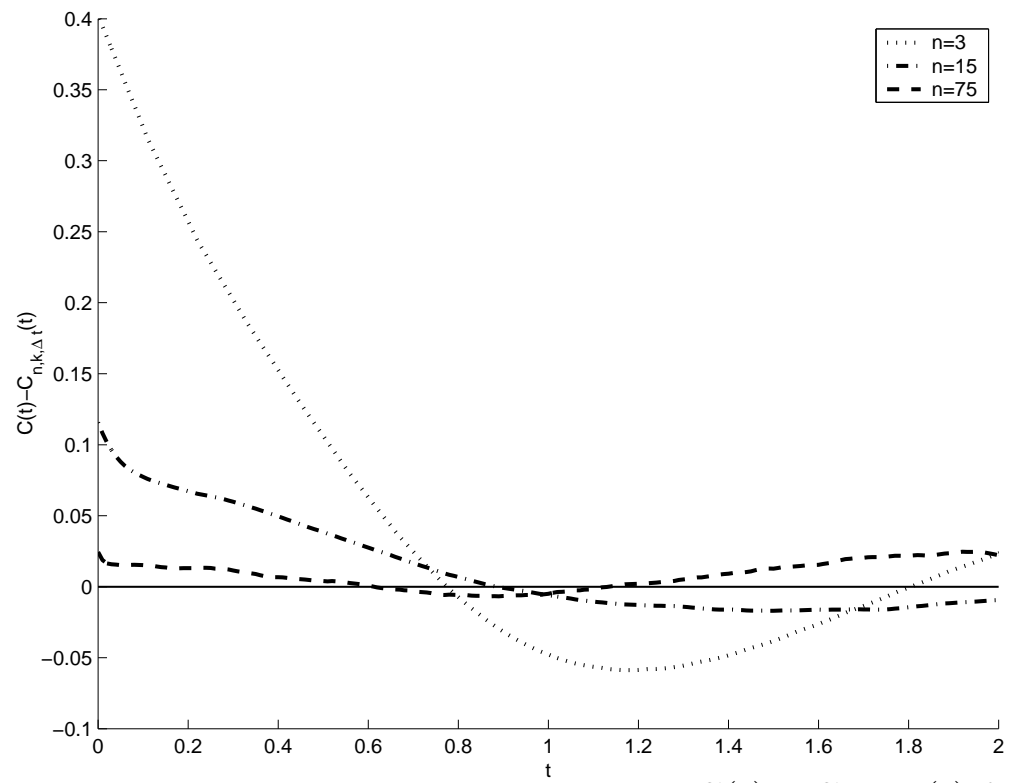

Figure 6.8: Projected symplectic Euler method. $C(t)-C_{n, k, \Delta t}(t)$ for $t \in[0,2]$ with $k=n^{2}$ and $\Delta t=1 / k$. The standard deviation of the error on the curves never exceeds 0.005 . 


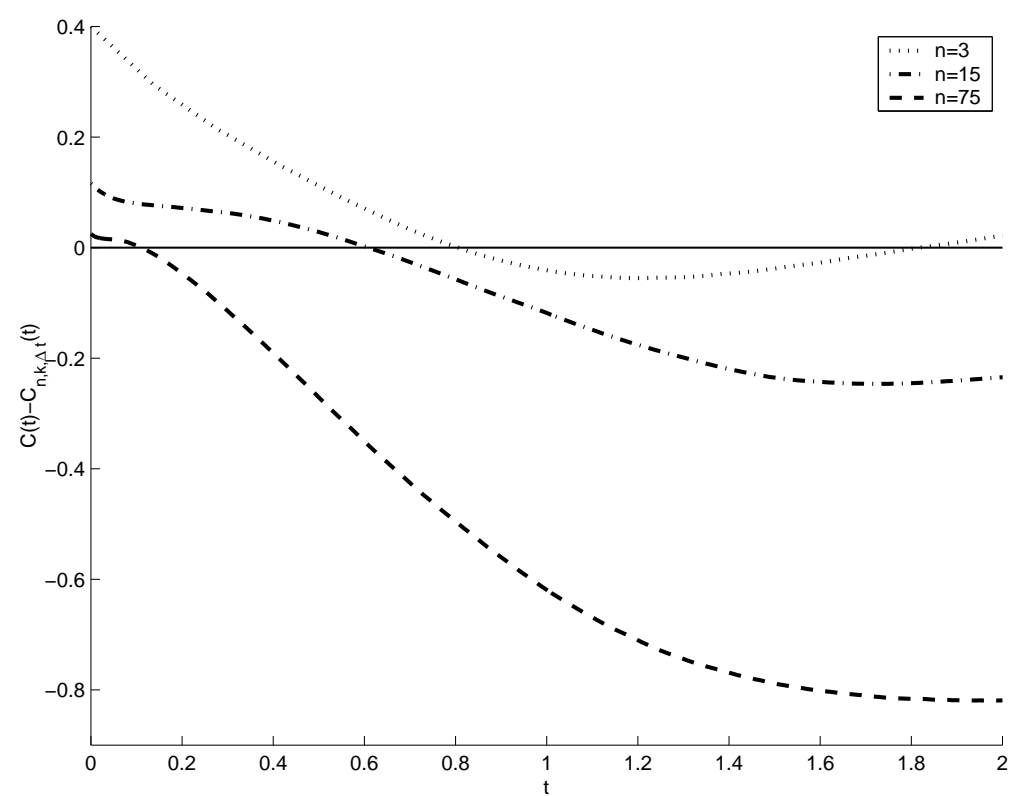

Figure 6.9: Projected symplectic Euler method. $C(t)-C_{n, k, \Delta t}(t)$ for $t \in[0,2]$ with $k=n^{2}$ and $\Delta t=1.251 / k$. The standard deviation of the error on the curves never exceeds 0.005 .

observed in individual trajectories previously.

Acknowledgments. This work was done while the author was supported by the Thomas V. Jones Stanford Graduate Fellowship. The author would like to thank Andrew Stuart for helpful discussions. Special thanks go to Jason Swanson for pointing out a serious error in an earlier draft of this manuscript.

\section{References}

[1] M. P. Allen and D. J. Tildesley, Computer Simulation of Liquids (Oxford University Press, Oxford, 1989).

[2] P. Billingsley, Convergence of Probability Measures, Second Edition (Wiley, New York, 1999). 
[3] B. Cano, A. M. Stuart, E. Süli, J. O. Warren, Stiff oscillatory systems, delta jumps and white noise, Found. Comput. Math. 1, 69-99 (2001).

[4] P. Deuflhard, J. Hermans, B. Leimkuhler, A. E. Mark, S. Reich, R. D. Skeel, Computational Molecular Dynamics: Challenges, Methods, Ideas (Lecture Notes in Computational Science and Engineering, Springer, Berlin, 1999).

[5] D. Dürr, S. Goldstein, J. L. Lebowitz, A mechanical model of Brownian motion, Comm. Math. Phys. 78, no. 4,507-530 (1980/81).

[6] R. Durrett, Probability: Theory and Examples, Second Edition (Duxbury Press, Belmont, Ca, 1996.)

[7] A. Dvoretzky, Asymptotic normality of sums of dependent random vectors, in Multivariate Analysis-IV, P. R. Krishnaiah, ed. (North Holland, 1977), pp. 23-34.

[8] G. W. Ford, M. Kac, On the quantum Langevin equation, J. Stat. Phys., 46:803-810, 1987.

[9] E. Hairer, C. Lubich, G. Wanner, Geometric Numerical Integration: Structure-Preserving Algorithms for Ordinary Differential Equations (Springer, Berlin, 2002).

[10] E. Hairer, S. P. Nørsett, G. Wanner, Solving Ordinary Differential Equations I: Nonstiff Problems (Springer, Berlin, 1987).

[11] O. H. Hald, R. Kupferman, Asymptotic and numerical analyses for models of heat baths, J. Stat. Phys., 106:1121-1184, 2002.

[12] T. E. Harris, Diffusion with "collisions" between particles, J. Appl. Prob. 2, 323-338 (1965).

[13] R. Holley, The motion of a heavy particle in an infinite one dimensional gas of hard spheres, ZWVG 17:181-219 (1971).

[14] W. Huisinga, C. Schütte, A. M. Stuart, Extracting macroscopic stochastic dynamics: model problems. Comm. Pure Appl. Math., 56, 234-269 (2003). 
[15] R. Kupferman, A. M. Stuart, J. R. Terry, P. F. Tupper, Long-term behaviour of large mechanical systems with random initial data. Stoch. Dyn. 2, 533-562 (2002).

[16] M. G. Mürmann, A semi-Markovian model for the Brownian motion, Séminar de Probabilité, VII, Lecture Notes in Math., Vol 321, Springer, 1973, pp. 248-272.

[17] L. Paoli, M. Schatzman, Vibrations avec contraintes unilatérales et perte d'énergie aux impacts, en dimension finie. C. R. Acad. Sci. Paris Sér. I Math. 317:97-101 (1993).

[18] R.-D. Reiss, Approximate Distributions of Order Statistics, Springer Series in Statistics (Springer-Verlag, New York, 1989).

[19] H. Sigurgeirsson, A. M. Stuart, Statistics from computations, in Foundations of Computational Mathematics (Oxford 1999), London Math. Soc. Lecture Note Ser. 284, Cambridge University Press, (Cambridge, 2001), pp. 323-344.

[20] F. Spitzer, Uniform Motion with Elastic Collision of an Infinite Particle System, J. of Math. and Mech. 18:973-989 (1968/1969).

[21] A. M. Stuart, J. O. Warren, Analysis and experiments for a computational model of a heat bath. J. Stat. Phys., 97:687-723, 1999.

[22] D. Turaev, V. Rom-Kedar, Elliptic islands appearing in near-ergodic flows. Nonlinearity, 11:575-600, 1998.

\section{A Appendix}

Recall the definition of $N_{n}$ from (3.9). We need to establish the following result: For any $t_{1}, t_{2}, \ldots, t_{d} \in[0, T]$ the random vector

$$
\left[N_{n}\left(t_{1}\right), N_{n}\left(t_{2}\right), \ldots, N_{n}\left(t_{d}\right)\right]
$$

converges in distribution to a Gaussian random vector with covariance matrix $\Sigma_{i j}=C\left(t_{i}-t_{j}\right)$. Here, $C(t)$ is as defined in (2.5). 
For our later convenience we define

$$
F_{t}\left(x_{1}, x_{2}\right):=\mathbb{P}\left\{y_{j}(0) \leq x_{1}, y_{j}(t) \leq x_{2}\right\}
$$

Since for each $j$ the process $y_{j}$ is stationary, this implies

$$
\mathbb{P}\left\{y_{j}\left(t_{1}\right) \leq x_{1}, y_{j}\left(t_{2}\right) \leq x_{2}\right\}=F_{t_{2}-t_{1}}\left(x_{1}, x_{2}\right)
$$

for any $t_{1}, t_{2} \in[0, T]$.

We apply a Central Limit Theorem of Dvoretzky ([7], Theorem 1). The result holds for certain dependent random vector arrays, but we will only need it for the independent case.

Theorem A.1 (From [7]). For each $n$, let $X_{n, i}, 1 \leq i \leq n$ be independent random column vectors in $\mathbb{R}^{d}$ with $\mathbb{E} X_{n, i}=0$. Let $\Sigma$ be a $d \times d$ matrix. For a vector $X$, denote its norm by $|X|$ and its transpose by $X^{T}$. For an event $A$, let

$$
\mathbb{E}(X ; A):=\mathbb{E}\left(X \mathbf{1}_{A}\right)
$$

Suppose

1. $\lim _{n \rightarrow \infty} \sum_{i=1}^{n} \mathbb{E} X_{n, i} X_{n, i}^{T}=\Sigma$,

2. for all $\epsilon>0, \lim _{n \rightarrow \infty} \sum_{i=1}^{n} \mathbb{E}\left(\left|X_{n, i}\right|^{2} ;\left|X_{n, i}\right|>\epsilon\right)=0$.

Then $S_{n}=X_{n, 1}+\cdots+X_{n, n} \Rightarrow \mathcal{N}(0, \Sigma)$ as $n \rightarrow \infty$.

In our case, $S_{n}$ and $X_{n, i}$ are vectors of length $d$ with $S_{n, j}=N_{n, j}$ and $X_{n, i, j}=n^{-1 / 2} \nu_{n, i, j}$, where $N_{n, j}$ and $\nu_{n, i, j}$ are defined in (3.9) and (3.8) respectively. We proceed to check the hypotheses of the CLT.

First note that $\mathbb{E} n^{-1 / 2} \nu_{n, i, j}=0$ for each $n, i, j$. Furthermore, with some algebra and the fact that

$$
\mathbb{E}\left[\mathbf{1}_{y_{i}\left(t_{j}\right) \leq n^{-1 / 2} x_{j}}\right]=\left(1+n^{-1 / 2} x_{j}\right) / 2,
$$


we obtain

$$
\begin{aligned}
& \sum_{i=1}^{n} \mathbb{E}\left[\left(n^{-1 / 2} \nu_{n, i, j}\right)\left(n^{-1 / 2} \nu_{n, i, k}\right)\right] \\
&= n^{-1} \sum_{i=1}^{n}\left[-\left(1+n^{-1 / 2} x_{j}\right)\left(1+n^{-1 / 2} x_{k}\right)\right. \\
&\left.\quad+4 \mathbb{P}\left\{y_{i}\left(t_{j}\right) \leq n^{-1 / 2} x_{j}, y_{j}\left(t_{k}\right) \leq n^{-1 / 2} x_{k}\right\}\right] \\
&= 4 F_{t_{k}-t_{j}}\left(n^{-1 / 2} x_{j}, n^{-1 / 2} x_{k}\right)-\left(1+n^{-1 / 2} x_{j}\right)\left(1+n^{-1 / 2} x_{k}\right)
\end{aligned}
$$

where $F_{t}$ is defined in (A.16). It can be shown that $F_{t}$ is continuous at $(0,0)$ for all $t \in[0, T]$, (for example, by showing that $F_{t}$ is Lipschitz in each variable there.) Hence the above quantity converges to $4 F_{t_{k}-t_{j}}(0,0)-1$ as $n \rightarrow \infty$. Lemma A.2 shows that $4 F_{t}(0,0)-1=C(t)$ for $t \geq 0$ so this is the appropriate covariance as stated in the theorem.

In order to verify the final condition of the CLT theorem, observe that $\left|\nu_{n, i, j}\right| \leq 2$ for all $n, i, j$, so

$$
\begin{aligned}
& \sum_{i=1}^{n} \mathbb{E}\left[\left|\left(n^{-1 / 2} \nu_{n, i, j}\right)\left(n^{-1 / 2} \nu_{n, i, k}\right)\right| ; \sum_{l=1}^{d}\left|n^{-1 / 2} \nu_{n, i, l}\right|>\epsilon\right] \\
& =\sum_{i=1}^{n} n^{-1} \mathbb{E}\left[\left|\nu_{n, i, j}\right|\left|\nu_{n, i, k}\right| ; \sum_{l=1}^{d}\left|\nu_{n, i, l}\right|>n^{1 / 2} \epsilon\right] \\
& \leq \mathbb{E}\left[4 ; 2 d>n^{1 / 2} \epsilon\right]=4 \mathbb{P}\left\{2 d>n^{1 / 2} \epsilon\right\}
\end{aligned}
$$

which converges to zero as required.

Lemma A.2 We have

$$
4 F_{t}(0,0)-1=C(t)
$$

Proof We can rewrite $F_{t}$ as

$$
\begin{aligned}
F_{t}\left(z_{1}, z_{2}\right) & =\mathbb{E}\left[\mathbf{1}_{q \leq z_{1}} \mathbf{1}_{G(q+t p) \leq z_{2}}\right] \\
& =\frac{1}{2} \int_{-\infty}^{\infty} \int_{-1}^{1} \mathbf{1}_{q \leq z_{1}} \mathbf{1}_{G(q+p t) \leq z_{2}} d q f(p) d p .
\end{aligned}
$$


So

$$
\begin{aligned}
4 F_{t}(0,0)-1 & =2 \int_{-\infty}^{\infty} \int_{-1}^{1} \mathbf{1}_{q \leq 0} \mathbf{1}_{G(q+p t) \leq 0} d q f(p) d p-1 \\
& =4 \int_{-\infty}^{\infty} \tilde{H}(p t) f(p) d p-1
\end{aligned}
$$

where we have defined

$$
\tilde{H}(z):=\frac{1}{2} \int_{-1}^{1} \mathbf{1}_{q \leq 0} \mathbf{1}_{G(q+z) \leq 0} d q .
$$

In order to obtain a more explicit expression for $\tilde{H}$, we first observe that it will be periodic with a period of 4 . Then some lengthy but straightforward calculations will show that

$$
\tilde{H}(z)=\left\{\begin{aligned}
(1-z) / 2, & 0 \leq z \leq 1 \\
0, & 1 \leq z \leq 2, \\
(z-2) / 2, & 2 \leq z \leq 3 \\
1 / 2, & 3 \leq z \leq 4
\end{aligned}\right.
$$

We can take advantage of the fact that $f$ is symmetric about 0 :

$$
\begin{aligned}
4 F_{t}(0,0)-1 & =4 \int_{-\infty}^{\infty} \tilde{H}(p t)(f(p)+f(-p)) / 2 d p-1 \\
& =2 \int_{-\infty}^{\infty}(\tilde{H}(p t)+\tilde{H}(-p t)) f(p) d p-1 \\
& =\int_{-\infty}^{\infty}[2(\tilde{H}(p t)+\tilde{H}(-p t))-1] f(p) d p
\end{aligned}
$$

The expression in the square brackets happens to equal $H$ of equation (2.4). So

$$
4 F_{t}(0,0)-1=\int_{-\infty}^{\infty} H(p t) f(p) d p=C(t)
$$

as required. 